Pacific Northwest

National Laboratory

Operated by Battelle for the

U.S. Department of Energy

\title{
Long Wave Infrared Cavity Enhanced Sensors
}

\author{
M. S. Taubman \\ P. M. Aker \\ D. C. Scott \\ M. D. Wojcik \\ B. D. Cannon \\ J. T. Munley \\ T. L. Myers \\ V. T. Nguyen \\ C. A. Bonebrake \\ J. F. Schultz
}

October 2004

Prepared for the U.S. Department of Energy under Contract DE-AC05-76RL01830 


\title{
DISCLAIMER
}

This report was prepared as an account of work sponsored by an agency of the United States Government. Neither the United States Government nor any agency thereof, nor Battelle Memorial Institute, nor any of their employees, makes any warranty, express or implied, or assumes any legal liability or responsibility for the accuracy, completeness, or usefulness of any information, apparatus, product, or process disclosed, or represents that its use would not infringe privately owned rights. Reference herein to any specific commercial product, process, or service by trade name, trademark, manufacturer, or otherwise does not necessarily constitute or imply its endorsement, recommendation, or favoring by the United States Government or any agency thereof, or Battelle Memorial Institute. The views and opinions of authors expressed herein do not necessarily state or reflect those of the United States Government or any agency thereof.

\author{
PACIFIC NORTHWEST NATIONAL LABORATORY \\ operated by \\ BATTELLE \\ for the \\ UNITED STATES DEPARTMENT OF ENERGY \\ under Contract DE-AC05-76RL01830
}

Printed in the United States of America
Available to DOE and DOE contractors from the Office of Scientific and Technical Information,
P.O. Box 62, Oak Ridge, TN 37831-0062;
ph: (865) 576-8401
fax: $(865) 576-5728$
email: reports@adonis.osti.gov

\footnotetext{
Available to the public from the National Technical Information Service, U.S. Department of Commerce, 5285 Port Royal Rd., Springfield, VA 22161 ph: (800) 553-6847 fax: $(703) 605-6900$ email: orders@ntis.fedworld.gov online ordering: http://www.ntis.gov/ordering.htm
}

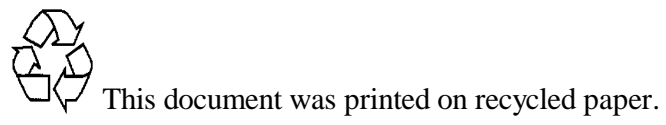




\title{
Long Wave Infrared Cavity Enhanced Sensors
}

\author{
M. S. Taubman \\ D. C. Scott \\ B. D. Cannon \\ T. L. Myers \\ C. A. Bonebrake \\ P. M. Aker \\ M. D. Wojcik \\ J. T. Munley \\ V. T. Nguyen \\ J. F. Schultz
}

October 2004

Prepared for

the U.S. Department of Energy under Contract DE-AC05-76RLO1830

Pacific Northwest National Laboratory

Richland, Washington 99352 


\section{Summary}

The principal goal of Pacific Northwest National Laboratory's (PNNL's) long wave infrared (LWIR) cavity enhanced sensor (CES) project is to explore ultra-sensitive spectroscopic techniques and apply them to the development of LWIR chemical sensors needed for detecting weapons proliferation. This includes detecting not only the weapons of mass destruction (WMDs) themselves, but also signatures of their production and/or detonation. The LWIR CES project is concerned exclusively with developing point sensors; other portions of PNNL's IR Sensors program address stand off detection. PNNL's LWIR CES research is distinguished from that done by others by the use quantum cascade lasers (QCLs) as the light source. QCLs are novel devices, and a significant fraction of our research has been devoted to developing the procedures and hardware required to implement them most effectively for chemical sensing. This report details the progress we have made on our LWIR CES sensor development.

During FY02, PNNL investigated three LWIR CES implementations beginning with the easiest to implement, direct cavity-enhanced detection (simple CES), including a technique of intermediate difficulty, cavity-dithered phase-sensitive detection (FM recovery CES) through to the most complex technique, that of resonant sideband cavity-enhanced detection also known as noise-immune cavityenhanced optical heterodyne molecular spectroscopy, or NICE-OHMS. This latter technique proved the most sensitive, with confirmed values of $9.7 \times 10^{-11} \mathrm{~cm}^{-1}(\mathrm{~Hz})^{-1 / 2}$ being observed, which is within a factor of 20 of the theoretical shot noise limit of $4.9 \times 10^{-12} \mathrm{~cm}^{-1}(\mathrm{~Hz})^{-1 / 2}$.

After attempts to improve instrument sensitivity in FY03, the optimum architecture for a fieldable LWIR CES became clear. Problems with reproducibly modulating the QCLs at the high frequencies necessary for NICE-OHMS, and the accompanying reduction in detector sensitivity at these frequencies, indicated that lower frequency operation was desirable. This caused us to choose the intermediate technique of the FM recovery over the resonant sideband technique of NICE-OHMS for the near term. In FY02 and FY03, we also explored the use of the sub-Doppler features known as Lamb dips, in order to increase both sensitivity and selectivity. At the low pressures required for the observation of such features however (10 milliTorr or less), typical LWIR-absorbing analytes saturate heavily even at the moderate operating powers experienced inside the optical cavities employed in the current experiments, resulting in loss of signal and thus sensitivity. Operation at higher pressures (1 to 10 Torr) reduces these saturation effects, by redistributing the upper and lower populations via collisional broadening. However, this also washes out any narrow features such as Lamb dips, indicating that Doppler-limited spectroscopy was the best recourse when operating at these higher pressures. The resulting sensor choice was the intermediate technique of the FM recovery CES, operating in the Doppler-limited regime at moderate pressures of a few Torr or so. This technique offers considerable sensitivity gains over the simple CES, but does not suffer from the complexity and fine tuning requirements of the full NICE-OHMS technique.

FY04 has seen extensive planning and infrastructure development necessary for the experimental work using the FM recovery architecture. For this second phase of LWIR cavity-enhanced experimental work, an entirely separate experiment is being constructed. Considerable effort has been invested in selecting test gases suitable both for laboratory and field experiments. This has involved selection of specific lines, sufficiently free of interference from ubiquitous atmospheric components such as water and 
carbon dioxide. As some of these selected gases are hazardous, the laboratory has been modified to ensure no threat is posed to its occupants. Once the molecular transitions were selected, suitable lasers were ordered from our supplier, Maxion technologies. Improvements have also been made to existing components, including laser mounting and related vacuum systems and laser drive electronics. 


\section{Contents}

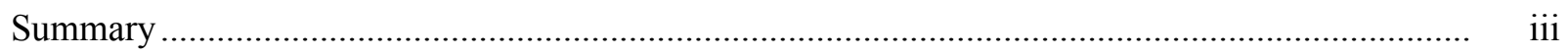

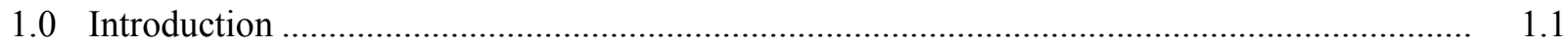

1.1 Summary of Investigated Sensors ...................................................................... 1.1

1.2 Discussion of FY03 Conclusions .............................................................................. 1.4

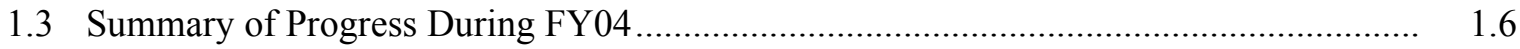

2.0 Selection of Test Molecules and Laser Wavelengths .................................................. 2.1

2.1 Summary of Test Molecules and Laser Wavelengths ................................................ 2.1

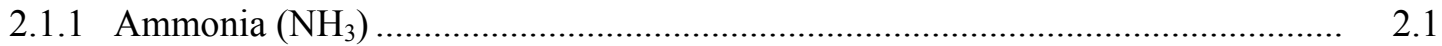

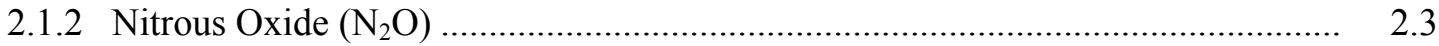

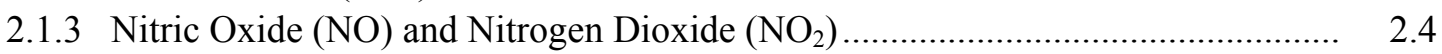

2.2 Summary of Selected Laser Lines ............................................................................... 2.5

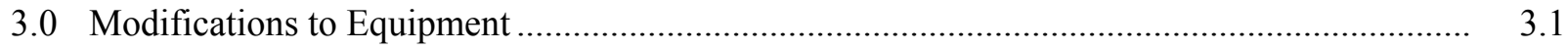

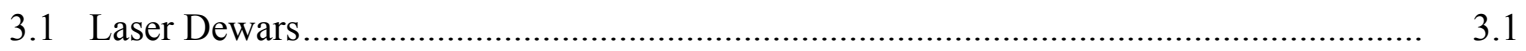

3.2 Laser Mounts .......................................................................................................... 3.4

3.3 Vacuum Systems, Manifolds, and Purging Requirements ........................................... 3.6

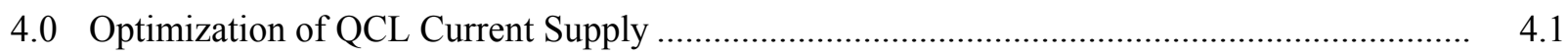

4.1 Stability of Main Regulator and Cascode Blocks …...................................................... 4.2

4.2 Noise of Cascode Blocks, a Question of Which Transistor .............................................. 4.3

4.3 Long-term Stability and Reference Drift................................................................... 4.5

4.4 Output Filtering, Resulting Resonances ................................................................ 4.5

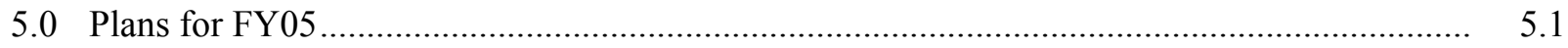

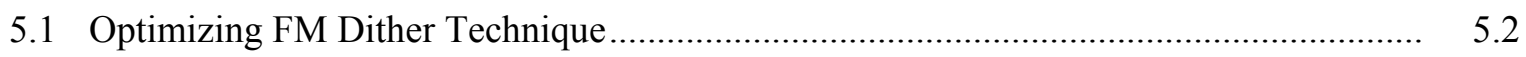

5.2 The Next Step in Stabilization .............................................................................. 5.2

5.3 Reducing the Footprint, and the Option of a Ring Cavity ........................................... 5.3

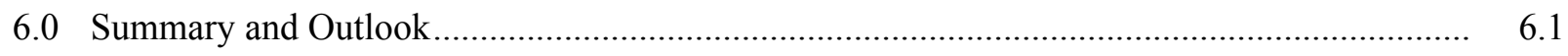

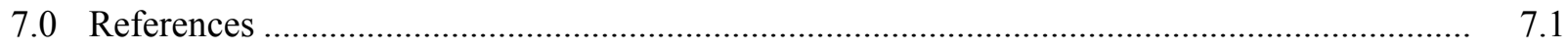




\section{Figures}

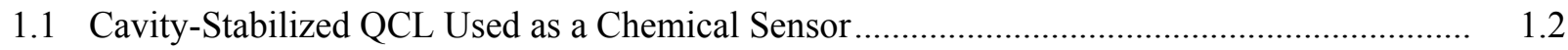

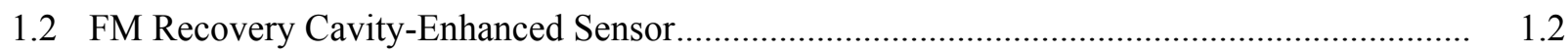

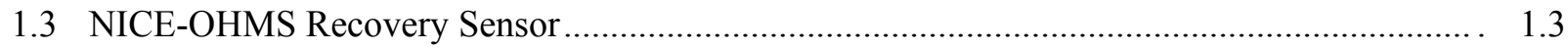

1.4 Sequence of Intra-Cavity Lamb Dips for Various Saturation Levels .................................. 1.5

2.1 Spectrum of Ammonia Comparing SWIR and LWIR Absorption Levels .......................... 2.2

2.2a Choice of Specific Ammonia Line by FM Dial Researchers at PNNL. .............................. 2.2

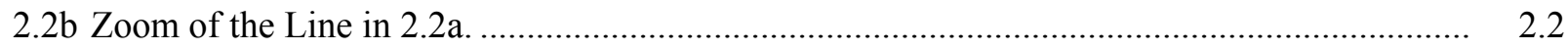

2.3a Spectrum of $\mathrm{N}_{2} \mathrm{O}$ P-Branch at $1271 \mathrm{~cm}^{-1}$, Showing Water Interference .............................. 2.4

2.3b Zoom of a Specific Component of Spectrum Shown in 2.4a, clear of Water Lines................ 2.4

2.4a Spectrum of $\mathrm{N}_{2} \mathrm{O}$ P-Branch at $1271 \mathrm{~cm}^{-1}$, Showing Lack of Water Interference. ................... 2.4

2.4b Zoom of a Specific Component of Spectrum Shown in 2.6a, Clear of Water Lines................ 2.4

3.1a QCL Cryogenic Laser Dewar Made by K-Dell for Laser Components. ................................. 3.1

3.1b Cutaway View of Laser Dewar Shown in Figure 3.1a................................................... 3.1

3.2a K-Dell Dewar Modified by PNNL Showing Custom Extended Outer Housing..................... 3.3

3.2b Cutaway View of the PNNL-Modified K-Dell Dewar..................................................... 3.3

3.3 Photograph of the Modified Dewar Components Before Assembly …................................ 3.4

3.4 View of K-Dell Dewar with Laser Mounted to Cold Plate ................................................ 3.5

3.5 New QCL Mount, Allowing Better Access and Thermal Conduction ................................ 3.6

3.6 Mixing Manifold Installed on the LeyBold Pumping Station ........................................... 3.7

4.1 Simplified Schematic of the QCL Current Controller ..................................................... 4.2 
4.2a Current Noise Comparison of Transistors 2N3904 and 2N2222A .................................... 4.4

4.2b Voltage Noise Comparison of Transistors 2N3904 and 2N2222A ........................................ 4.4

4.3a Voltage Noise Levels in Cascode Circuit Employing 2N3904 Transistor ............................ 4.5

4.3b Voltage Noise Levels in Cascode Circuit Employing 2N2222A Transistor .......................... 4.5

4.4 Transfer Function of Fast Input Network ..............................................................

5.1 FM Recovery Cavity-Enhanced Sensor showing Areas of Focus ..........................................

5.2 Cut-away Views of the Original Piezo Mount ............................................................. 5.2

5.3 Cut-away Views of Modified Piezo Mount................................................................. 5.3

5.4 Ring Cavity Version of the FM Recovery Sensor. ......................................................... 5.4

\section{Table}

2.1 Summary of Chosen Laser Frequencies and Wavelengths............................................. 2.5 


\subsection{Introduction}

Various CES architectures were examined during FY02 and FY03. Various operational limitations were discussed in detail in the FY03 report. The brief summary of these architectures given in the FY03 report is reproduced here for the readers' convenience, and then the findings in the FY03 report are summarized.

\subsection{Summary of Investigated Sensors}

The most basic cavity-enhanced sensor we tested is shown in Figure 1.1. It is referred to as the "Simple Cavity-Enhanced Sensor", since it is based on direct transmission through the optical cavity, without the use of any modulation technique other than that involved in locking the laser to the optical cavity. The optical field from a quantum cascade laser (QCL) is coupled into an optical cavity via an acousto-optic modulator (AOM) and a Faraday isolator in order to minimize the optical feedback into the QCL from back reflections. Reflected and transmitted light from the cavity are observed using detectors D1 and D2 respectively. The QCL is locked to the optical cavity using the Pound-Drever-Hall (PDH) technique, the electronics for this being compressed into one block called "stabilization electronics." The optical cavity also forms a chamber allowing an analyte to be introduced at low pressures. A piezoelectric element in contact with the transmission mirror allows the optical cavity length to be scanned, thus changing the cavity mode frequencies and that of the QCL since it is locked to one of these modes. As the frequency of the sensor is scanned, the signal from detector D2, is filtered at some bandwidth B, measured on an oscilloscope and then recorded.

The advantages of this sensor are that its operation is relatively simple, and it gives a direct result that can exactly be modeled mathematically. Because of this, it is an extremely useful tool for measuring absorption and saturation values of various test analytes. The disadvantages are that the sensitivity is directly limited by the low frequency amplitude noise of the laser and detector, specifically, "1-over-f" or $1 /$ f noise.

The next kind of sensor we use is the "FM Recovery Cavity-Enhanced Sensor", shown in Figure 1.2. Here, a modulation technique is added to reduce the impact of the low frequency noise mentioned above. Again, the QCL is locked to the peak of the cavity mode and the cavity length is scanned or swept to move both mode and laser frequency across molecular absorption features. The difference is that in addition to be being scanned, the cavity length is also rapidly "dithered" using a modulation frequency in the $10 \mathrm{~s}$ of $\mathrm{kHz}$. This cavity modulation signal (independent of that applied to the laser in order to lock it to the cavity), is applied directly to the piezoelectric element in addition to the signal used to produce the frequency sweep. Since this modulation frequency is well within the bandwidth of the laser-locking loop, the laser frequency follows this cavity dither in the same way as it follows a cavity sweep. The signal from the transmission detector is then demodulated at this dither frequency, after which, it is filtered and recorded on a digital oscilloscope as before. 
SIMPLE CAVITY-ENHANCED SENSOR

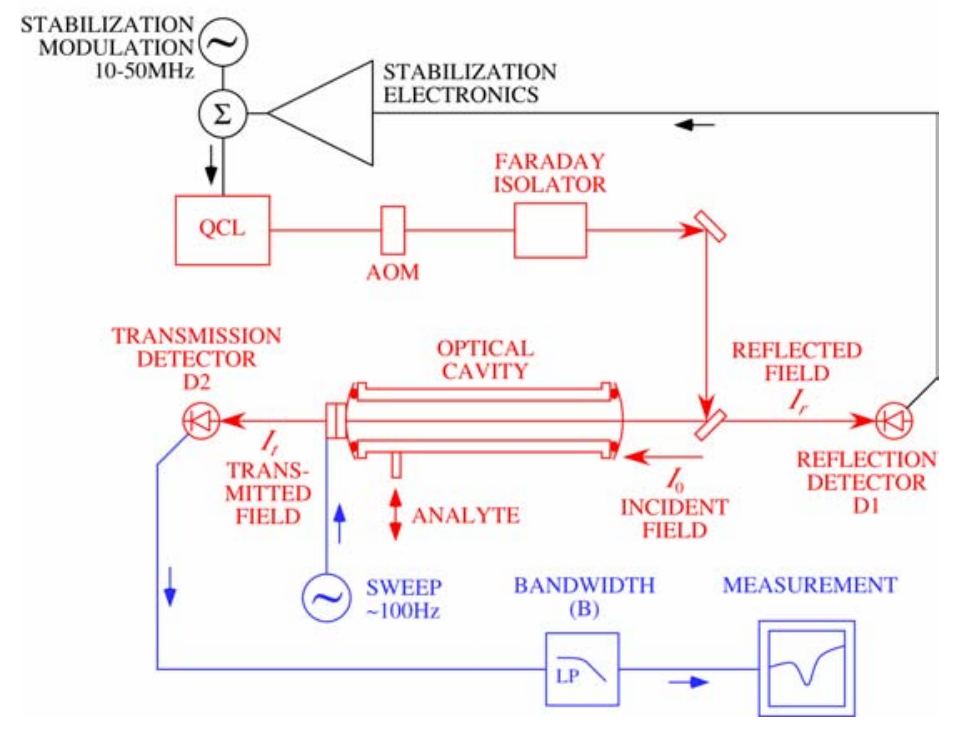

Figure 1.1. Cavity-Stabilized QCL Used as a Chemical Sensor. Absorption due to the intra-cavity analyte causes dramatic changes in the cavity transmission. Optical elements are in red, stabilization in black, measurement electronics in blue. Incident, reflected and transmitted cavity fields of intensities $I_{0}, I_{r}$ and $I_{t}$ are indicated.

FM RECOVERY CAVITY-ENHANCED SENSOR

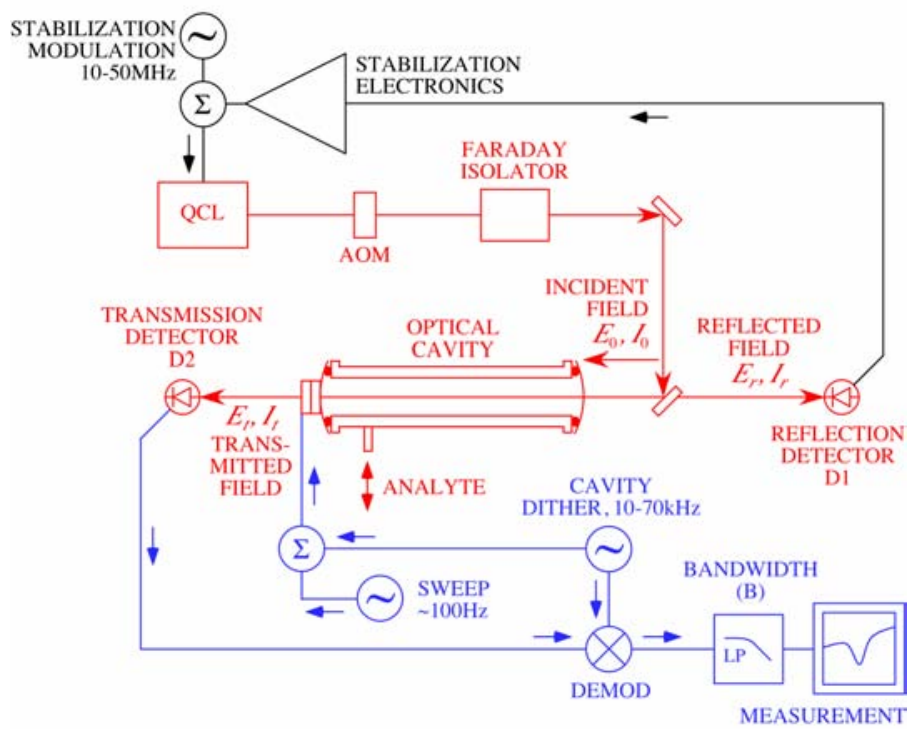

Figure 1.2. FM Recovery Cavity-Enhanced Sensor. The QCL is locked to the cavity as before.

However, in addition to the cavity length being scanned, it is also dithered or modulated. The transmission signal from D2 is demodulated accordingly, then filtered and recorded as before. Intensities and electric field amplitudes are indicated for incident, reflected and transmitted fields. 
The main advantage of this dithered cavity sensor is that the cavity dither encodes the absorption information onto the light at a frequency above the regions where $1 / \mathrm{f}$ noise dominate, thus increasing the resulting signal to noise of the sensor. Demodulation of the signal seen at detector D2 at this cavity modulation frequency then decodes the information, and derivative-like absorption features can be obtained. Another advantage is that this technique is sensitive to spectral features of a specific width, and hence the modulation depth can be adjusted to target certain features.

The disadvantage of this sensor is a slight increase in complexity. The modulation technique requires a source, the piezoelectric behavior needs to be understood, and the sensor needs to be more carefully optimized. However, these disadvantages are relatively minor in comparison to the benefits of this sensor.

The next step in the development of cavity-enhanced sensors was to use the technique of resonant sideband detection, or NICE-OHMS (Ye, Ma, and Hall 1998; Gianfrani, Fox, and Hollberg 1999; Ishibashi and Sasada 1999). Figure 1.3 shows the NICE-OHMS experimental arrangement. In addition to the modulation required for the locking of the QCL to the optical cavity, a modulation is applied at the cavity free spectral range (FSR - the frequency interval between consecutive cavity modes), which was 387.5 MHz for these experiments. The resulting sidebands on the incident field coincide exactly with separate optical cavity modes and thus enter the optical cavity in addition to the carrier. The field transmitted from the cavity is detected and demodulated first at this high frequency, and then at the

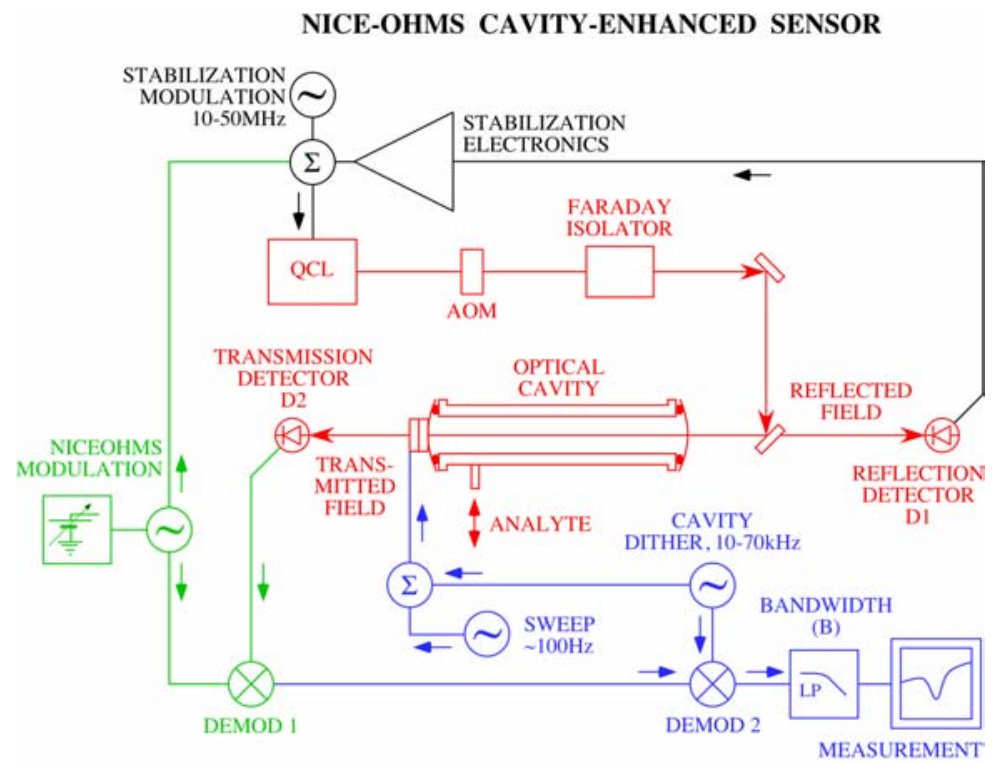

Figure 1.3. NICE-OHMS Recovery Sensor. A high frequency modulation is imposed on the laser in addition to that required for the cavity locking. This high frequency is equal to the spacing between adjacent cavity modes. The resulting transmission signal is first demodulated at this frequency, then secondly at the cavity modulation frequency as explained for the FM recovery cavity-enhanced sensor. 
cavity-dither modulation frequency, these processes being represented by DEMOD1 and DEMOD2 respectively. The resulting signal is then filtered at some bandwidth and monitored during cavity scans as before.

The NICE-OHMS sensor configuration shown here potentially has the best performance of all the presented techniques. To begin with, the resonant sideband technique gives immunity to noise in the lock between the QCL and the optical cavity, becoming more important if cavities with very high finesse are used, which are harder to lock a laser to. The high frequency modulation required to achieve NICEOHMS results in the FM detection occurring at a frequency where the technical noise on the laser is virtually non-existent, giving the best possible immunity to $1 / \mathrm{f}$ noise. The cavity-dither technique also employed here allows the selectivity of the instrument to be increased as previously discussed. The result is an instrument with highly optimized sensitivity and selectivity.

While this technique promises a lot, it is considerably more complex. It requires careful matching between cavity length and modulation frequency, which, while not being so extreme as to require an automated system, can be a problem at first (or any subsequent recalibration), because no signal is seen until the matching is reasonably close. Also, the detectors available at these wavelengths do not perform well at high frequencies resulting in considerable loss of signal, and the modulation characteristics of QCLs at these frequencies have not been found to be uniform.

\subsection{Discussion of FY03 Conclusions}

During FY03, several limitations were discovered pertaining both to the use of high frequency modulation and detection in the LWIR with QCL-based CES, and the use of sub-Doppler spectroscopic techniques in high finesse optical cavities. While not so severe that they justify halting research into these areas, it was clear that they limit the applicability of both the NICE-OHMS technique and the use of subDoppler features for the development of fieldable sensors in the LWIR at this time. For this reason the techniques of direct cavity transmission detection and Doppler-limited FM recovery, using Dopplerlimited spectroscopy, were chosen as viable fieldable technologies.

The first and most profound limitation was that of saturation when observing the sub-Doppler features known as Lamb dips, as shown in Figure 1.4. When molecular transitions are pumped hard enough so that the excitation rate is faster than the thermalization rates via collisions, spontaneous emission and other processes, the upper levels become significantly populated. This causes stimulated emission to compete with the absorption process, and causes the effective absorption of the medium to decrease. At low levels, this results in the appearance of sub-Doppler features, which in principle offer increased selectivity and sensitivity. At high levels however, the result can be a large reduction in absorption, and thus signal and sensitivity. At an extreme, it can even result in complete transparency of the medium. To understand saturation and sub-Doppler features, we consider the thermal distribution of velocities along the longitudinal axis of the optical cavity. Molecules with different velocities interact with the optical field at different frequencies due to the effect of the Doppler shift. This gives rise to a Gaussian broadening of spectral features, known as the "Doppler profile". Also, molecules moving with some speed along this axis interact only with the optical field propagating in one direction: the Dopplershifted frequency of the counter-propagating field is too far detuned. However, molecules with relatively 


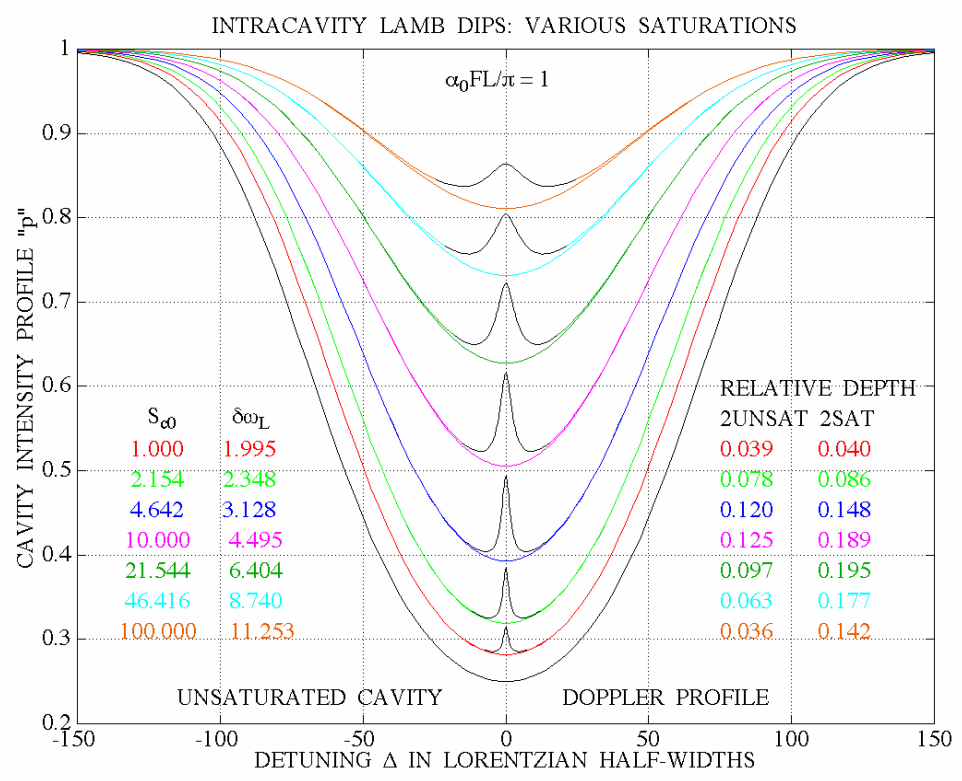

Figure 1.4. A Sequence of Intra-Cavity Lamb Dips Solved Using an Iterative Numerical Technique. The relative Lamb dip size reaches a maximum and then decreases. For this calculation, the absorption strength is roughly the same as the other cavity losses; expressed by the condition $\alpha_{0} F L / \pi=1$, where $\alpha_{0}, F$ and $L$ are unsaturated absorption coefficient, cavity finesse and length respectively.

small longitudinal velocities interact with both fields simultaneously. Being partially saturated by one field, the resulting absorption of the other is reduced, resulting in a dip with the shape of the homogeneously broadened line, far narrower than the Doppler line.

Figure 1.4 shows the effects on a spectral profile of saturation at various levels. For reference, an unsaturated profile is shown in black, being the deepest absorption feature. As the level of saturation $S_{c 0}$ increases, the Lamb dip depth increases to a maximum and then slowly decreases, both relative to the unsaturated and saturated Doppler profiles (labeled "RELATIVE DEPTH, 2UNSAT and 2SAT" in the annotation). The width of the Lamb dip also increases, shown here in relative line-width units, $\delta \omega_{L}$. However, the depth of the Doppler feature also decreases as saturation increases, resulting in less overall absorption.

As cavity finesse is increased in order to improve sensitivity, intra-cavity power easily becomes high enough to saturate typical LWIR small-molecule analytes. The resulting reduction in absorption clearly limits the effective sensitivity of the instrument. Our chosen method to alleviate this problem is to increase the sample detection pressure. Lamb dips appear at a particular set of conditions, and require low pressures such that collisional broadening is less that of the homogenous line-width. This unfortunately directly contributes to the saturation issue, as little will depopulate the relatively long-lived upper states. Increasing the pressure, depopulates these upper states alleviating saturation and deepening the Doppler absorption profiles, but washes out the Lamb dips. 
The second limitation, which in fact compounds the first, is that we are heavily detector noise-limited, i.e., the dominant noise source is the detection process. This is especially true at high detection frequencies where the responsivity of the mercury-cadmium-telluride (MCT) detectors we use roll off to $35 \mathrm{~dB}$ less than the typical DC values. This drastically reduces the possible signal-to-noise ratios using the NICE-OHMS technique, and renders it approximately equivalent in sensitivity to our other best techniques. In addition, this also implies that we cannot reduce the impact of the saturation issue described above by reducing the power circulating in the cavity, as any gains in absorption sensitivity would be wiped out by decreasing signal-to-noise at the detector.

The third limitation involves the logistics and the modulation characteristics of the QCL. The NICEOHMS technique requires the period of the RF modulation frequency to match the round-trip time of the optical cavity, which would require an optical cavity longer than $1.5 \mathrm{~m}$ to match a modulation frequency of less than $100 \mathrm{MHz}$. The requirement for logistically tolerable cavity lengths forces the use of modulation frequencies greater than $100 \mathrm{MHz}$. In the frequency range above $100 \mathrm{MHz}$, the QCL frequency modulation characteristics are poor and vary significantly from device to device. In this frequency range, adequate frequency modulation for NICE-OHMS is only possible at a peak in the FM characteristics. This peak is also narrow. Use of a cavity that was too long by $0.5 \mathrm{~cm}$ and the required modulation frequency change caused a difference in signal-to-noise ratio of 10 or more. It has also been demonstrated that the optimal modulation frequency is different from laser to laser. This clearly makes building a sensor based on this technique difficult. External modulation techniques have already been demonstrated impractical as the resulting devices finish by being larger and more power hungry than the others that we are already trying to miniaturize for the purposes of making fieldable equipment.

In light of these limitations our choice of architecture to proceed in the development of a fieldable sensor is either direct cavity transmission technique (the "Simple Cavity-Enhanced Sensor"), or the cavity-dither FM recovery technique, and target Doppler-limited spectroscopic features rather than subDoppler features. With the use of Doppler-limited features and operation with buffer gas pressures $\sim 10$ Torr, saturation should not be a problem, and cavity-enhanced sensors of high finesse should be able to operate successfully. High frequency modulation and response issues of both the QCLs and the MCT detectors are avoided, since the system will operate at tens of kilohertz rather than hundreds of megahertz. The detector elements can be larger, greatly alleviating the additional issues of alignment and optical fringing, discussed in the FY03 report.

\subsection{Summary of Progress During FY04}

The selection of a specific sensor architecture represents a new phase of experimental research and development. Until now, the focus of the experimental work was to demonstrate and explore the performance of the various architectures, and to identify and quantify the limitations of each technique. This was done without the need to select specific target chemicals, and consequently analytes were chosen more for convenience, ease of use and safety rather than for strategic importance. A major part of the work conducted in FY04 was to choose more strategic analytes, and molecular transitions.

Implementing better gas handling systems and modifying laboratory systems to accommodate these analytes was also part of this work. In addition, a number of minor but time consuming technical issues 
important for fieldable sensor design, but not critical for the first experimental phase, were delayed until this point. These included improving the alignment stability and mounting system of the QCL within the cryogenic dewar, and refining the drive electronics for the QCL. These are the issues we addressed during FY04, and will be discussed in detail in this report. 


\subsection{Selection of Test Molecules and Laser Wavelengths}

The method for selecting suitable molecular species and transitions has been an iterative process involving our QCL supplier Maxion Technologies. Due to the large number of wavelength possibilities, in order expedite laser manufacture and delivery, it was necessary to streamline our QCL requirements by coordinating wavelength choices with other projects.

\subsection{Selection of Test Molecules and Laser Wavelengths}

In this section we discuss some of our choices for test molecules. Two database resources were used for this research, the first being Molspec, based on the Hitran database. The test conditions for spectra obtained from Molspec in this report were chosen to be 10 Torr analyte pressure corresponding to projected fieldable sensor operating pressure, a temperature of $296 \mathrm{~K}$, and over a path length of $16 \mathrm{~cm}$ as a desirable target cell length. Analyte concentration was chosen to be 1 part per million (ppm) as a standard test sample concentration. Standard impurities were chosen to include water at $1 \%$, corresponding to a relative humidity of $35 \%$, and carbon dioxide at $500 \mathrm{ppm}$. The second database used for this analysis is that being compiled by Steve Sharpe, the conditions for which are 1ppm-meter at 296K, taken at 1 atmosphere. All specified wavelengths are specified in vacuum.

\subsubsection{Ammonia $\left(\mathrm{NH}_{3}\right)$}

Ammonia has been the chosen analyte of the short-wave infrared (SWIR) sensor being developed at PNNL by Richard Williams because it challenges gas-handling systems, and is representative of the physical and spectral characteristics of large categories of chemicals. Ammonia is a sticky molecule, and as such is typical of many analytes of interest, and demands that certain parts of the system be passivated with a glass layer in order to minimize absorption into the walls, and thus shorten both response and recovery times of the apparatus. We also chose ammonia in order to demonstrate the benefit of going to the LWIR due to the vastly increased absorption cross section at 9.5 micron as compared to 1.5 micron where the SWIR system operates. This is illustrated by the spectrum in Figure 2.1.

Warren Harper, who leads PNNL's FM Dial experiments, has extensively searched for an ammonia line clear of water interference, and has chosen $1046.39 \mathrm{~cm}^{-1}(9.557 \mu \mathrm{m})$. We will also use this line in order to utilize the same laser design implemented by Maxion, and facilitate rapid delivery. This chosen line is shown in Figure 2.2, ammonia lines being shown in magenta, and water lines in blue.

Note the considerable structure shown in the zoom of the ammonia line shown in Figure 2.2b. This structure makes the line easier to identify. 


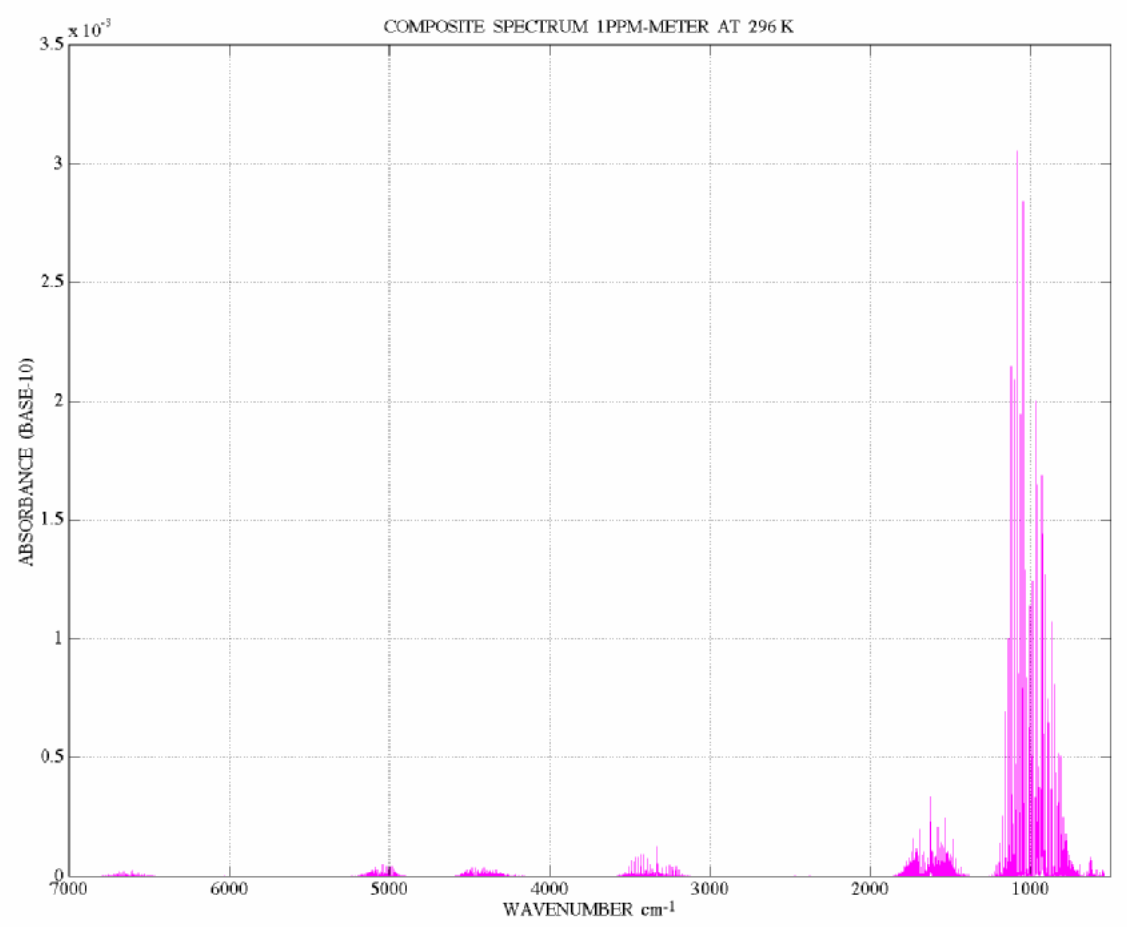

Figure 2.1. Spectrum of Ammonia Showing the Difference in Absorbance from the SWIR through to the LWIR

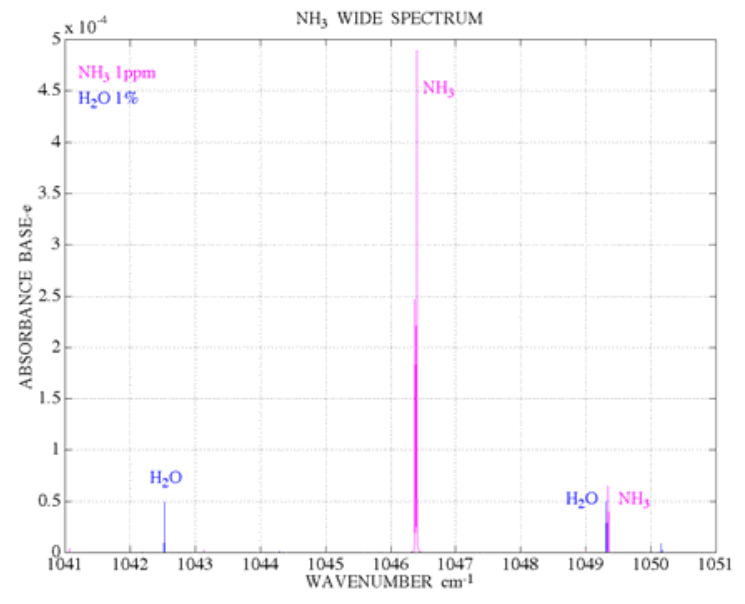

Figure 2.2a. FM Dial Choice of $\mathrm{NH}_{3}$ Line at $1046.39 \mathrm{~cm}^{-1}$. Water interference lines in blue far from the line.

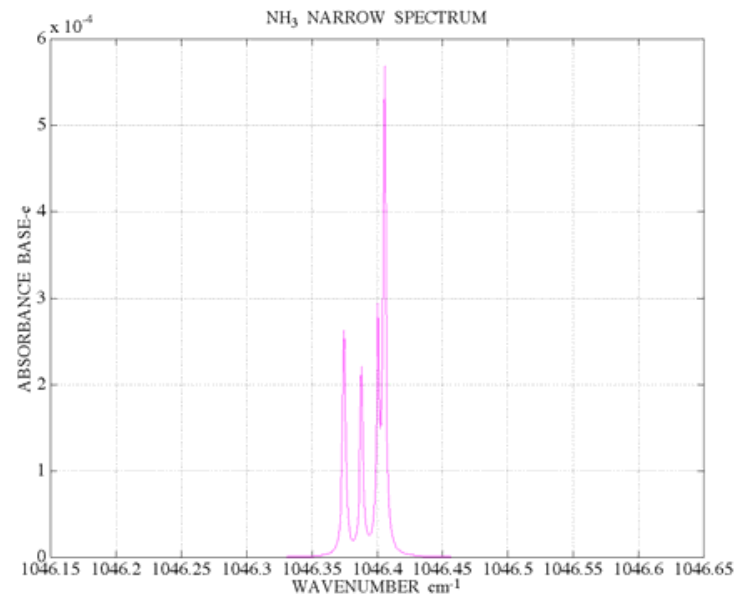

Figure 2.2b. A Zoom of this Line. Note the structure - useful as a signature. 


\subsubsection{Nitrous Oxide $\left(\mathrm{N}_{2} \mathrm{O}\right)$}

Nitrous oxide was our previous choice for the initial tests of the LWIR chemical sensor architectures, which were performed at around $1170 \mathrm{~cm}^{-1}$, or 8.5 micron. The reason for this is that it is non-toxic, noncorrosive, easy to obtain and convenient to use. It is also an explosives decomposition product, and an industrial breakdown product. $\mathrm{N}_{2} \mathrm{O}$ is present in the atmosphere at about 325 parts per billion (ppb), but is a convenient analyte, with good absorption at a range of wavelengths. In broadening our QCL-based sensor development to cover more wavelengths, new systems will be built using different optics and detectors, for example, InSb instead of MCT. Nitrous oxide will allow us to test these new sensors easily and safely. One advantage of using an analyte that is present in the atmosphere at low levels is that once a sensor is operating at high enough sensitivities to reliably detect atmospheric $\mathrm{N}_{2} \mathrm{O}$, continuous testing can be done without the need for a test gas release. This is also of significant interest to the development of remote sensors at PNNL, as well as point sensors.

We chose nitrous oxide wavelengths in the $\mathrm{P}$ - and $\mathrm{R}$-branches between 1230 to $1330 \mathrm{~cm}^{-1}$ ( $\left.7.8 \mu \mathrm{m}\right)$, and in the stronger $\mathrm{P}$ and $\mathrm{R}$-branches between 2170 and $2270 \mathrm{~cm}^{-1}$. The transitions from 1230 to $1330 \mathrm{~cm}^{-1}$ fall in the 5 to $8 \mu \mathrm{m}$ region where water lines limit atmospheric transmission. This brings us to a significant advantage of point sensors over remote sensors. The capability of working at low pressures allows us to remove the effects of atmospheric pressure broadening and obtain potentially better resolution and selectivity. Because of this, we can choose lines that are too cluttered for remote sensors to use. Also, these lines are close enough to our previous operating values of $1170 \mathrm{~cm}^{-1}$ to allow the use of much of the same optics and similar MCT detectors, while allowing us to examine the effectiveness of operating in this region. The chosen lines are at $1271.077 \mathrm{~cm}^{-1}(7.8673 \mu \mathrm{m})$, and $1298.609 \mathrm{~cm}^{-1}$ $(7.7005 \mu \mathrm{m})$. The first is shown in Figures 2.3a and 2.3b, $\mathrm{N}_{2} \mathrm{O}$ lines shown in red, and water in blue. (The second is not shown.) Note that larger water lines have been clipped to allow the nitrous oxide lines to be clearly seen. Despite the presence of these large water lines, at the pressure of 10 Torr the narrow plots of the selected lines show we can avoid any significant water interference. There are small water lines further out than the range of the narrow plots up to $0.5 \mathrm{~cm}^{-1}$ away, but these will not present a problem either.

The transitions between 2170 and $2270 \mathrm{~cm}^{-1}$ (around $4.5 \mu \mathrm{m}$ ) are on the mid-wave side of the water transmission window, and the lines here are relatively unencumbered by water. However, they are in a significantly different region and experimental components including optics and detectors will have to be changed. We have chosen the region from $2193.5 \mathrm{~cm}^{-1}$ to $2208.1 \mathrm{~cm}^{-1}(4.54 \mu \mathrm{m})$, which can hopefully be covered by temperature tuning one laser. This region is shown in Figure 2.4a with a zoom of lines near $2208 \mathrm{~cm}^{-1}$ shown in Figure 2.4b. The absorption bands of the G-agent tabun discussed above are near $2193.5 \mathrm{~cm}^{-1}$. 


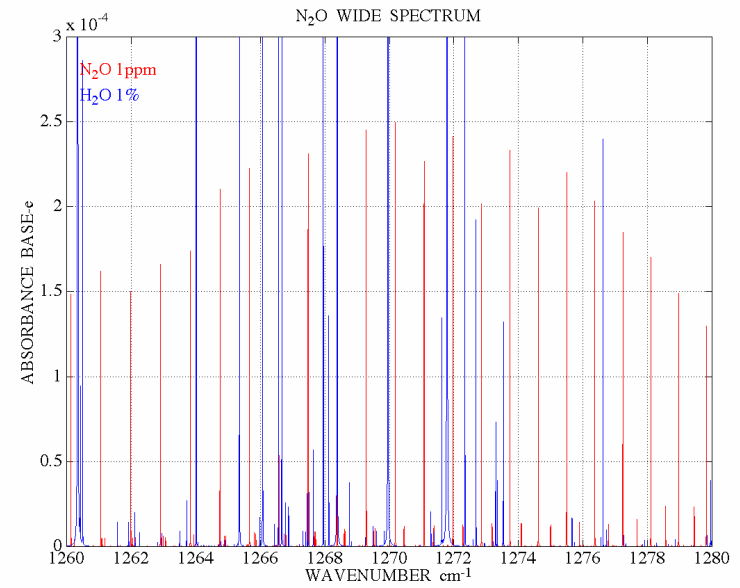

Figure 2.3a. $\mathrm{N}_{2} \mathrm{O} \mathrm{P}-\mathrm{Branch}$ Centered on $1270 \mathrm{~cm}^{-1}$. Selected line at $1271.077 \mathrm{~cm}^{-1}$, relatively clear of water.

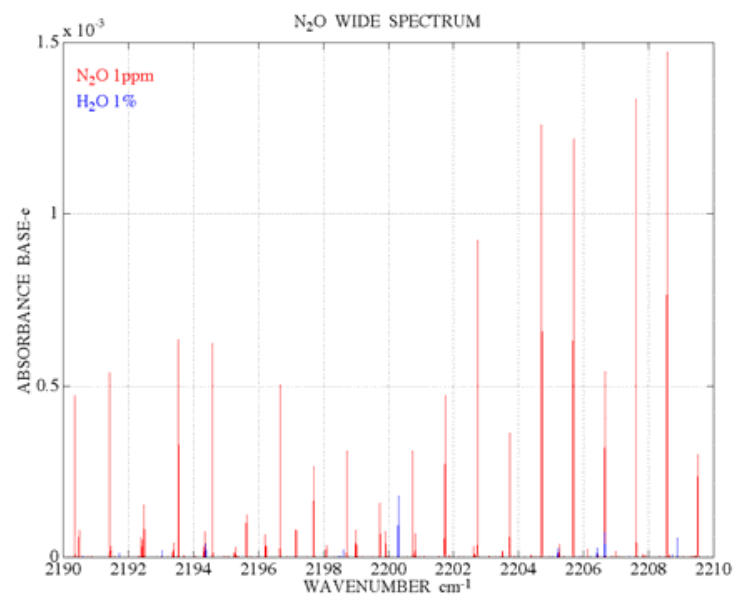

Figure 2.4a. Lower Frequency Half of $\mathrm{N}_{2} \mathrm{O}$ PBranch Centered on $2208 \mathrm{~cm}^{-1}$. Note by comparison to $1300 \mathrm{~cm}^{-1}$ bands, the relative lack of water lines.

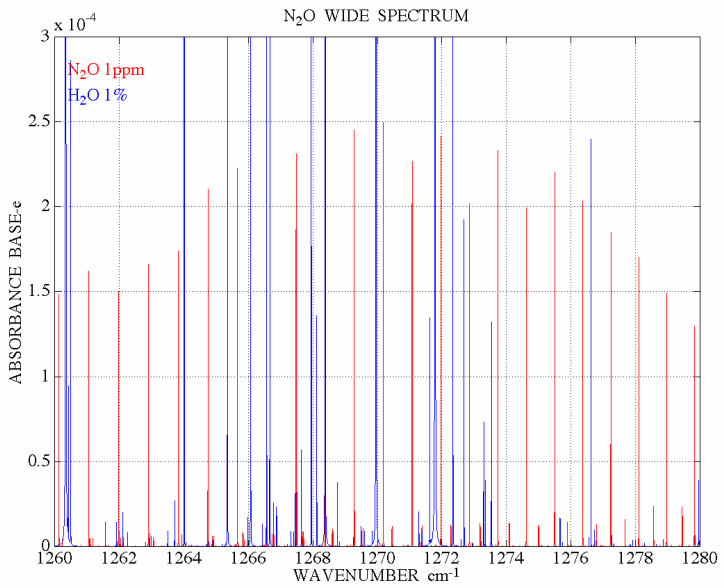

Figure 2.3b. Narrow Plot of $\mathrm{N}_{2} \mathrm{O}$ Line at $1271.077 \mathrm{~cm}^{-1}$. Small water lines nearby that won't interfere.

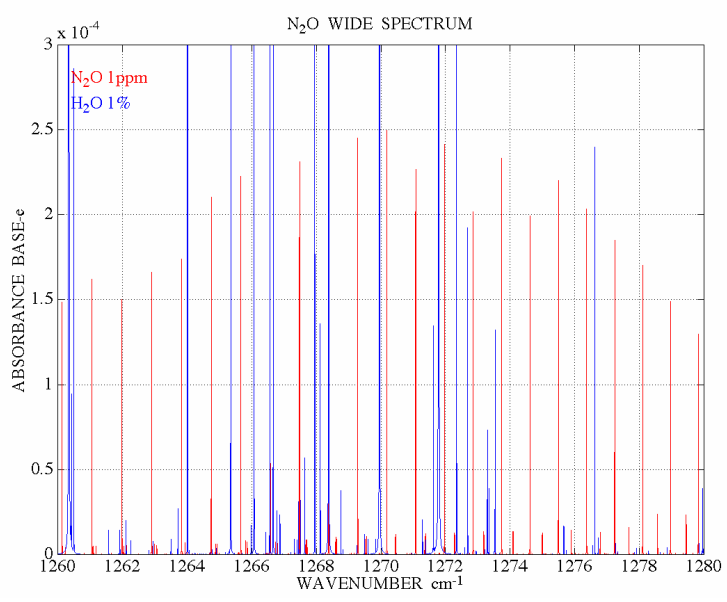

Figure 2.4b. Narrow Plot of $\mathrm{N}_{2} \mathrm{O}$ Lines Near $2208 \mathrm{~cm}^{-1}$. Small water lines nearby that won't interfere.

\subsubsection{Nitric Oxide (NO) and Nitrogen Dioxide $\left(\mathrm{NO}_{2}\right)$}

Both nitric oxide and nitrogen dioxide are byproducts of the processing of nuclear fuel rods, decomposition products of explosives, and also produced in other industrial processes. Nitrogen dioxide is of particular interest as a product of photolysis of TNT and other explosives. Both are reactive chemicals and some care must be taken to handle them in the laboratory, but in small quantities this will not present a problem. NO has $\mathrm{P}-$ and $\mathrm{R}-$ branches at 1800 to $1950 \mathrm{~cm}^{-1}$ with well spaced lines since it is 
a diatomic molecule. In $\mathrm{NO}_{2}$ we chose lines and groups of lines near the peaks of the $\mathrm{P}$ - and $\mathrm{R}$-branches of the band from 1560 to $1660 \mathrm{~cm}^{-1}$. We have found many possible lines in these two molecules, but will not discuss them all explicitly here.

\subsection{Summary of Selected Laser Lines}

A number of additional laser lines were chosen to permit assessment of clutter in spectral regions of operational interest. The table in Table 2.1 summarizes the clutter lines of interest as well as lines chosen for detection of test chemicals.

Table 2.1. Summary of Chosen Laser Frequencies and Wavelengths. Priority for each choice is also listed.

\begin{tabular}{|c|c|c||}
\hline Frequency, $\mathbf{c m}^{-\mathbf{1}}$ & Wavelength, $\boldsymbol{\mu m}$ & Priority \\
\hline 1046.3 & 9.557 & 1 \\
1084.6 & 9.220 & 1 \\
1271.077 & 7.8673 & 5 \\
1298.609 & 7.7005 & 5 \\
$2208.1 \mathrm{~T} \pm 0.5$ & 4.5288 & 5 \\
$1903.4 \mathrm{~T} \pm 0.4$ & 5.2538 & 7 \\
$1900.3 \mathrm{~T} \pm 0.4$ & 5.2623 & 7 \\
$1849.7 \mathrm{~T} \pm 0.6$ & 5.4063 & 7 \\
$1853.35 \mathrm{~T} \pm 0.6$ & 5.3956 & 7 \\
$1600.2 \mathrm{~T} \pm 0.6$ & 6.2492 & 6 \\
$1630.3 \mathrm{~T} \pm 0.3$ & 6.1339 & 6 \\
$1251.3 \mathrm{~T} \pm 0.7$ & 7.9917 & 2 \\
$1355.0 \mathrm{~T} \pm 1.0$ & 7.3801 & 4 \\
$1048.2 \mathrm{~T} \pm 0.8$ & 9.5402 & 3 \\
\hline
\end{tabular}




\subsection{Modifications to Equipment}

During FY04 time has been taken to modify and improve several aspects of the experimental apparatus used for the LWIR-CES experiments. These improvements are also beneficial to related projects using the same hardware, such as the remote FM Dial and laser development work. These modifications consist of a redesign of the cryogenic dewar in which the lasers are mounted, a redesign of the laser mounting system within the dewar, and a redesign of the QCL current supply. An innovation specific to the LWIR-CES was the design and construction of a mixing manifold, which facilitates easier mixing of analytes. These will be covered in detail in this section.

\subsection{Laser Dewars}

Although QCLs are now running routinely at room temperature in pulsed mode, for distributed feedback grating type QCLs running CW, cryogenic operation gives the most power and reliability. Early on in the project, it was found that by far the best dewar for this purpose was that made by K-Dell for Laser Components Inc, costing around $\$ 6 \mathrm{~K}$ each, represented in Figure 3.1.

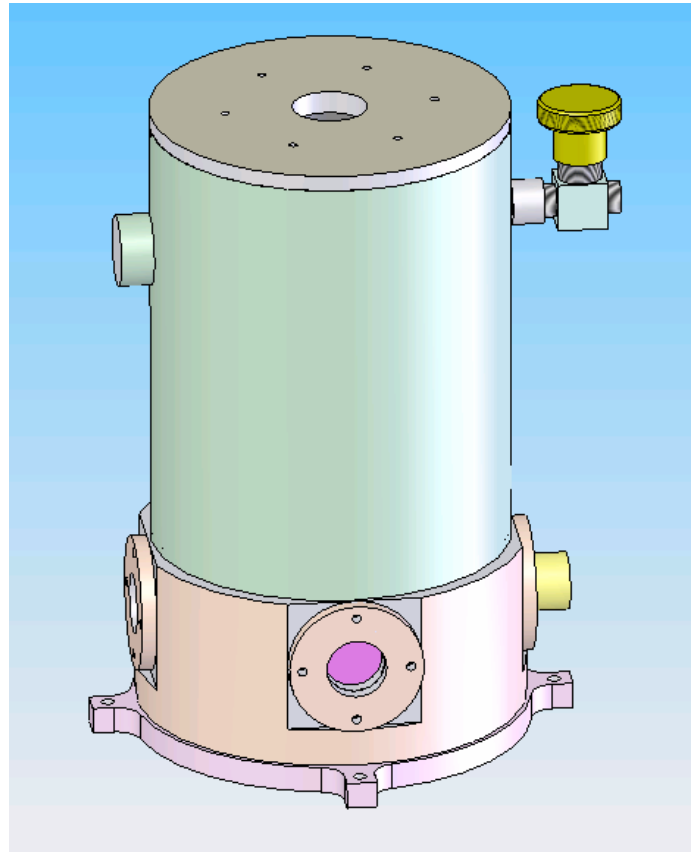

Figure 3.1a. QCL Cryogenic Laser Dewar Made by K-Dell for Laser Components, Showing Output Window (BaF), Vacuum Pumping Port at Top, and Electrical Connector Bottom Right

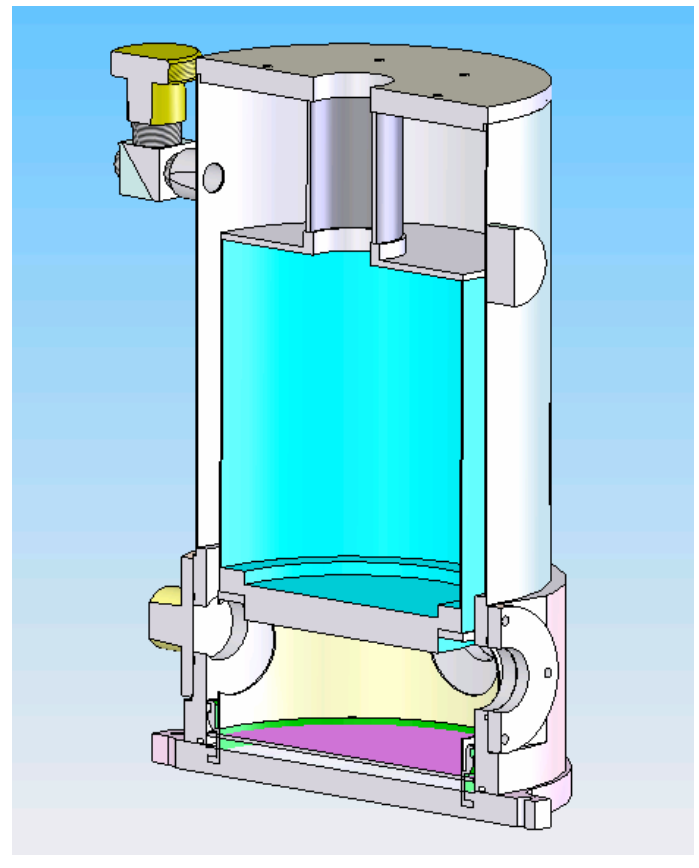

Figure 3.1b. Cutaway View of the Dewar, Showing the Filler Neck, $\mathrm{LN}_{2}$ Reservoir, and Cold Plate to which the Cold Finger is Attached. A large circular fiberglass fitting extends from the cold plate down to float above the dewar base plate. 
The dewars consist of an outer aluminum housing that is evacuated via a valve attached to the side, and an inner vessel shown in blue, which contains the $\mathrm{LN}_{2}$. At the top of this vessel, a fiberglass filler neck (dark gray) connects the vessel to the top of the outer housing using a standard epoxy (Armstrong, type A12). The base of the $\mathrm{LN}_{2}$ vessel is a thick piece of aluminum constituting the cold plate of the dewar, to which can be attached the dewar payload. A large circular fiberglass fitting is attached to the outside of the cold plate, and extends almost down to the dewar base plate, the purpose of which is presumably to help dampen pendulum motion of the $\mathrm{LN}_{2}$ reservoir.

These dewars have an $\mathrm{LN}_{2}$ hold time without any internal heat source of around three days, and with the QCL running (dissipating around five watts), of about six hours. They are well made, and more mechanically stable than any others used by members of our group. Nevertheless, mounting QCLs in dewars presents several challenges, including reduced access to the device, which must be run under vacuum in order to prevent condensation and freezing of water vapor on the laser surfaces. A resulting problem is difficulty in capturing the entire divergent output beam and collimating it, due to the distance between the QCL output facet and any lens one would place outside the dewar. The presence of the $\mathrm{BaF}$ window in between adds to scatter and back reflections. A considerable advance was made in FY02, whereby a specialized multi-element lens was designed and fabricated, and an assembly designed and built especially for these dewars, which entirely replaces the BaF window, allowing closer access to the QCL output facet. (This assembly is now manufactured and sold by High Precision Devices in Boulder Colorado.) These lenses and mounting systems provide superior collimation and output power coupling compared to the previous arrangement. However, an additional problem has arisen as a result. Because the lens is now much closer to the QCL than before, the pointing of the laser output beam is far more sensitive to movement of the laser mounting assembly. It was found that when cooling from room temperature down to cryogenic temperatures, there is a vertical movement in the laser mount assembly of approximately $0.8 \mathrm{~mm}$ relative to the dewar base plate, due to contraction of the $\mathrm{LN}_{2}$ vessel, which as discussed above, is fixed to the outer housing via the filler neck of the dewar, shown in Figure 3.1b. While small, this movement causes a vertical shift in the output beam position of $5 \mathrm{~cm}$ or more when observing the beam after several meters of propagation, corresponding to an angular change of about 10 degrees or so. The repeatability of the beam position at these distances is also poor when heating and cooling the dewar back down, which happens on a daily basis. Consequently, daily realignment of the laser system is required. Even during the course of a few hours, minor adjustments are required maintain good alignment of the cavity-locked sensor systems used in the LWIR-CES.

In order to alleviate this problem, the laser dewars are being modified to remove this internal movement as depicted in Figure 3.2. Firstly, the top of the dewar was machined off, and replaced with a specially extended version, shown here in purple. This allowed extra room at the top of the dewar, so that a set of stainless steel bellows could be attached above the filler neck using a specialized epoxy. The bellows were attached to the underside of the new top surface of the outer housing via a bolted flange and O-ring arrangement, providing a good vacuum seal. Thirdly, the dewar base plate was modified to accommodate an aluminum annulus, shown in green, which connects to the fiberglass insulator shown in light yellow, thus fixing the vertical position of the dewar cold plate to the base plate. Now, the bellows at the top of the dewar take up any vertical movement resulting from expansion or contraction of the $\mathrm{LN}_{2}$, and the cold plate remains fixed. 


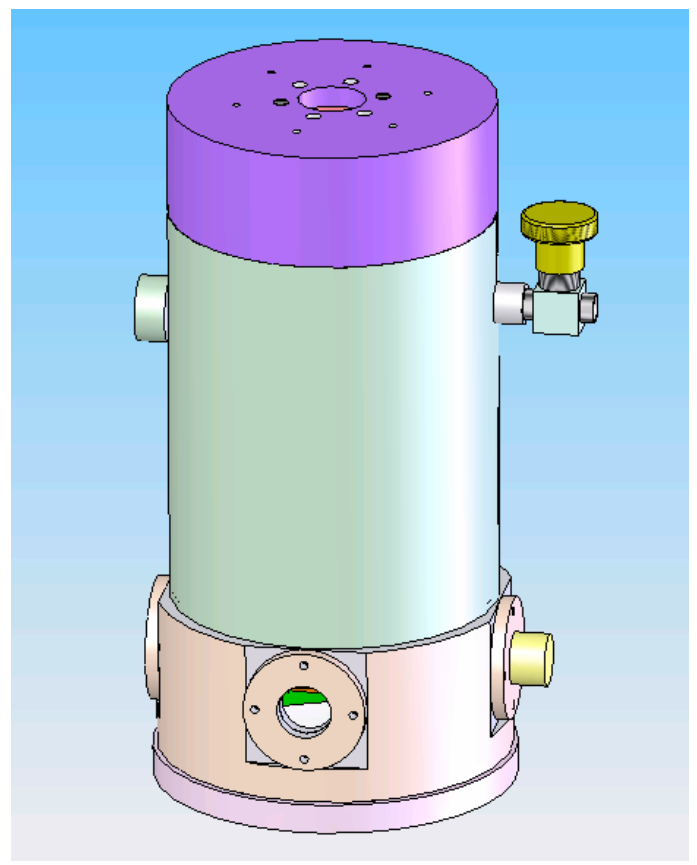

Figure 3.2a. Modified Dewar, Showing Custom Extended Outer Housing

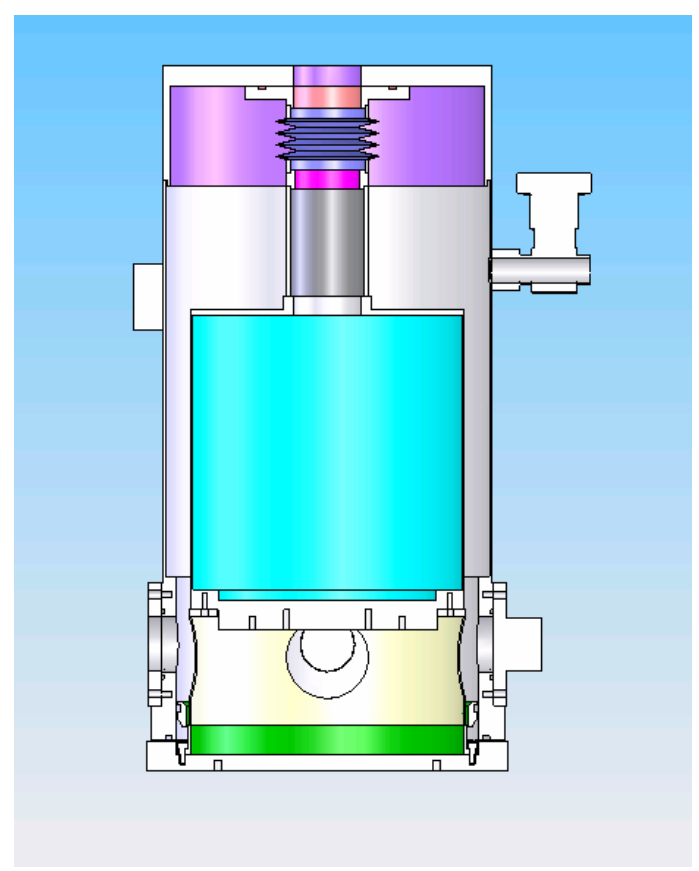

Figure 3.2b. Cutaway Version of the Modified Dewar, Showing Bellows at the Top, and a Rigid Mount at the Bottom

While relatively easy in concept, the addition of the bellows proved not to be an easy task. The epoxy joint between the bellows and the filler neck must be able to withstand vacuum conditions without significant out-gassing, and sustain continual daily cycling from room temperature to $77 \mathrm{~K}$ without cracking or leaking. In addition, bellows are generally not made from aluminum because they would weaken and break with vacuum cycling over time, and consequently are usually made from stainless steal. However, stainless steel is notoriously difficult to fasten using epoxy. Tests with the standard cryogenic epoxy used elsewhere in the dewar (Armstrong type A-12) showed that the joints leaked within one or two temperature cycles. A search of various specialty vendors has resulted in the acquisition of several types of space-rated epoxy. Currently, the tested epoxy is from Master Bond Inc, type "Supreme $10 \mathrm{HT}$ ", and tens of temperature cycles under vacuum has not shown any degradation in quality, the internal vacuum remaining at a very good $10^{-6}$ Torr. The reconstructed dewar is now ready for testing, and will be so early in FY05. More dewars will then be modified. The components of the modified dewar are shown below in Figure 3.3.

The question arises, since the addition of the bellows proved to be the difficult part of the modifications, what would happen had the laser mounting plate simply been secured to the dewar base plate without the addition of bellows at the top. The result of a simple calculation shows that the resulting internal force resulting from differential contraction during thermal cycling would be around $7500 \mathrm{lbs}$, or 33000 Newton's, the result being that any epoxy bonds holding the assembly together would be broken 


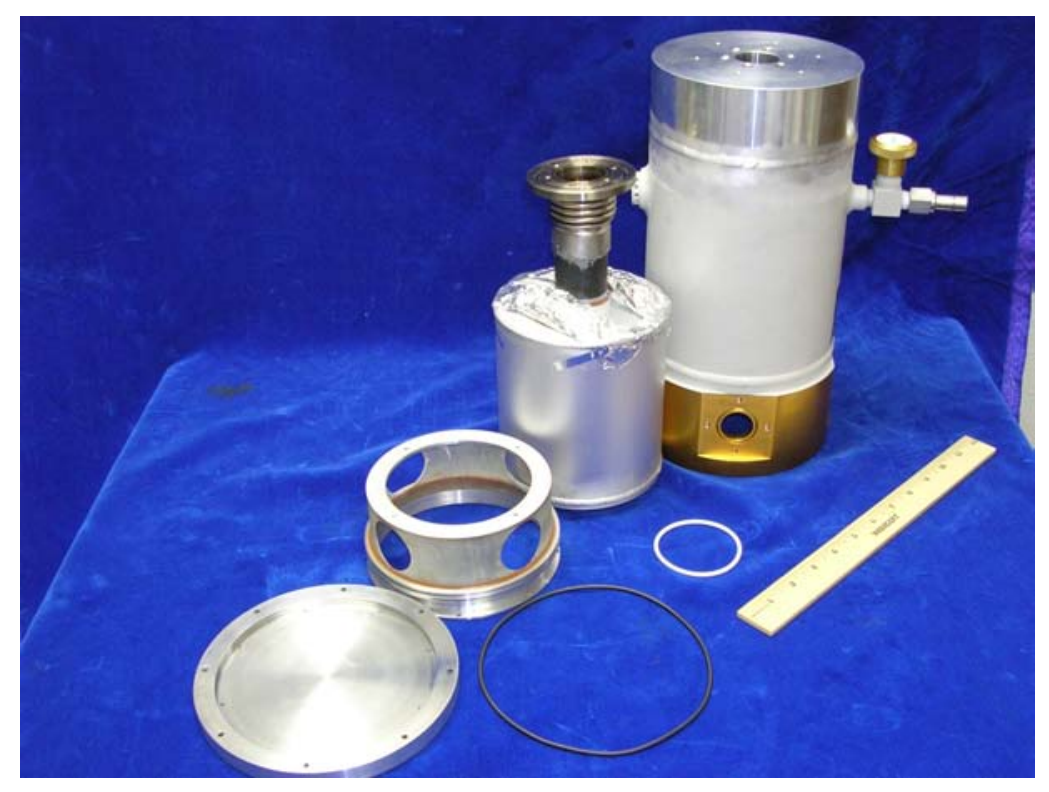

Figure 3.3. View of the Components of the Modified Laser Components Dewar Before Assembly

long before such a force was reached. Another question arises as to why these dewars weren't fitted with such a facility to eliminate such a problem. The answer is most likely that our work requires more precision than that for which most others employ these same dewars.

\subsection{Laser Mounts}

In addition to the above problems with movement of the cold plate, the actual mounting of the QCLs to the cold plate has been an on-going issue. The dewars are sold with a cold finger arrangement ready for mounting of payloads at the ends of the cold fingers next to the $\mathrm{BaF}$ windows in the side ports of the outer dewar housing. A picture of this arrangement is shown in Figure 3.4.

This is a view a K-Dell dewar with mounted QCL, standing upside-down on the table, with the base plate removed. The gold-colored cold finger is clearly visible, and the QCL is mounted on a copper piece clamped to the end of the finger, inside the recess to the upper right beyond which is the BaF window. A portion of the copper clamp and mounting screws are visible. The connecting wires for the QCL are also clearly seen, passing to vacuum rated SMA connectors on the opposite recess, top left. A heater and temperature sensor are placed in the center of the cold finger and the connecting wires going to the multipin connector shown bottom left. This allows the temperature of the cold finger to be raised slightly above $\mathrm{LN}_{2}$ temperature, which facilitates both temperature stabilization and temperature tuning of the payload mounted on the cold finger. In order to make this easier and more efficient, the dewars come standard with small Teflon washers paced between the cold finger and the dewar base plate, providing some degree of insulation between the two, thus facilitating temperature change. This was not a problem for the intended application of these dewars, which was for lead-salt lasers dissipating considerably less power than QCLs. However, in order to get adequate cooling with of the QCL from the dewar base plate, these Teflon washers had to be removed, rendering the temperature adjustment mechanism highly 
inefficient. This cold finger arrangement has been replaced with a custom-mounting bracket discussed below.

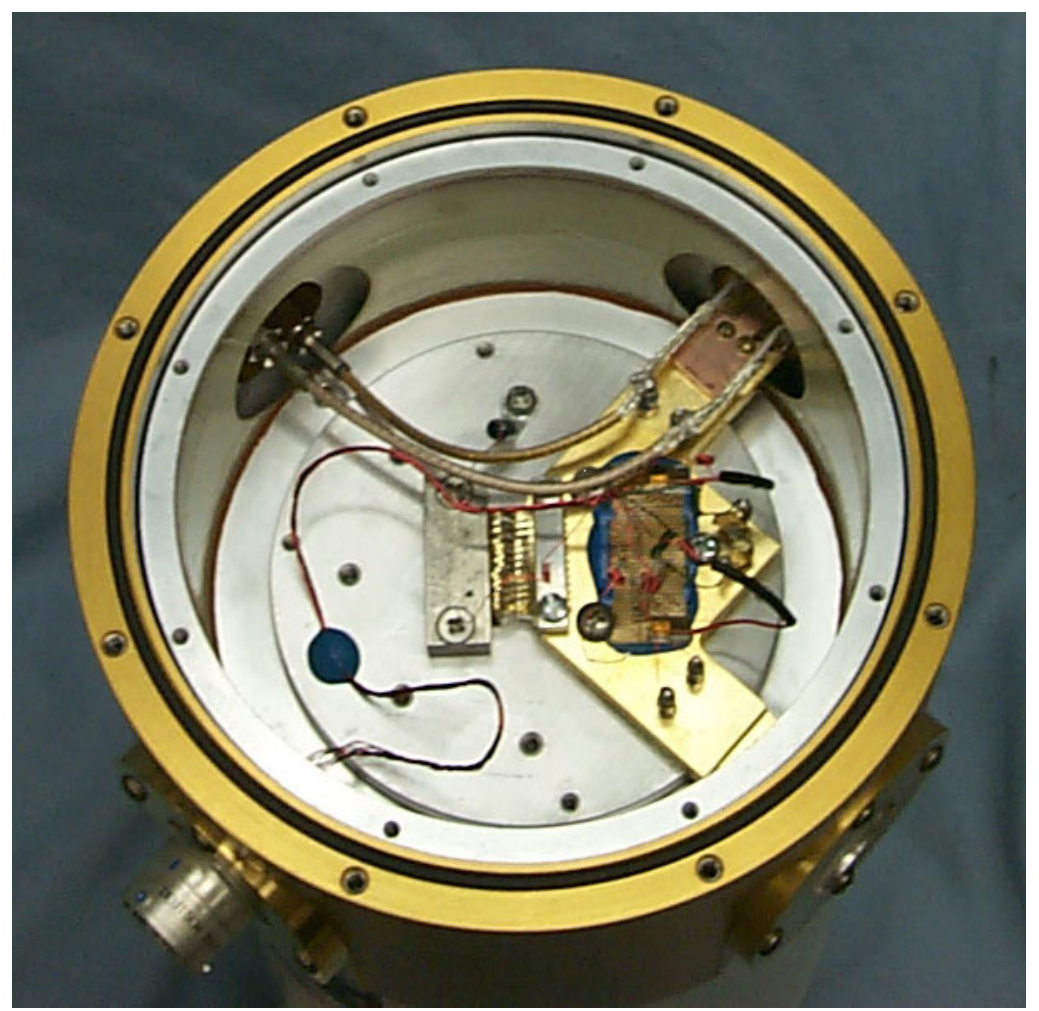

Figure 3.4. View of a K-Dell Dewar, Inverted, Base Plate Removed. The cold plate and gold-covered cold finger are clearly visible. QCL is mounted on cold finger in recessed port top right. SMA cables connect the QCLs to vacuum rated SMA bulkheads in recessed port top left. Heater and temperature sensor mounted in middle of cold finger connected to multi-pin connector bottom left.

Another related problem is the sub-mount upon which the QCL chip itself is mounted. Since QCLs have not yet been commercially standardized, they are often provided from different suppliers mounted in different ways on different mounts. The earlier mounted versions obtained from Lucent Technologies, required soldering of electrical contacts mounted on insulating pads glued down with either varnish or cryogenic epoxy, to which the wire bonds to the laser were connected. After approximately six months of operation, this arrangement became difficult to remove or alter, often resulting in destruction of the wire bonds, or worse, destruction of the laser device itself. To this end, the laser sub-mount has been redesigned to match exactly with that of ALPES Lasers in Switzerland, one of the major European suppliers from whom we have purchased QCLs and from whom we would be most likely to purchase from again other than Maxion Technologies. All lasers we use from this point on will be mounted on this laser sub-mount (including those purchased from Maxion Technologies), unless special requirements prevail. The new mounting bracket and laser sub-mount assembly is shown in Figure 3.5. The new mounting bracket is thicker, shorter and has larger contact area than the original cold finger, providing 
better thermal conduction between the QCL and the dewar base plate. In order to still obtain efficient temperature adjustment of the QCL, the heater (not shown) and sensor (dark gray on the side) were moved to the end of the mounting bracket where the QCL is situated.

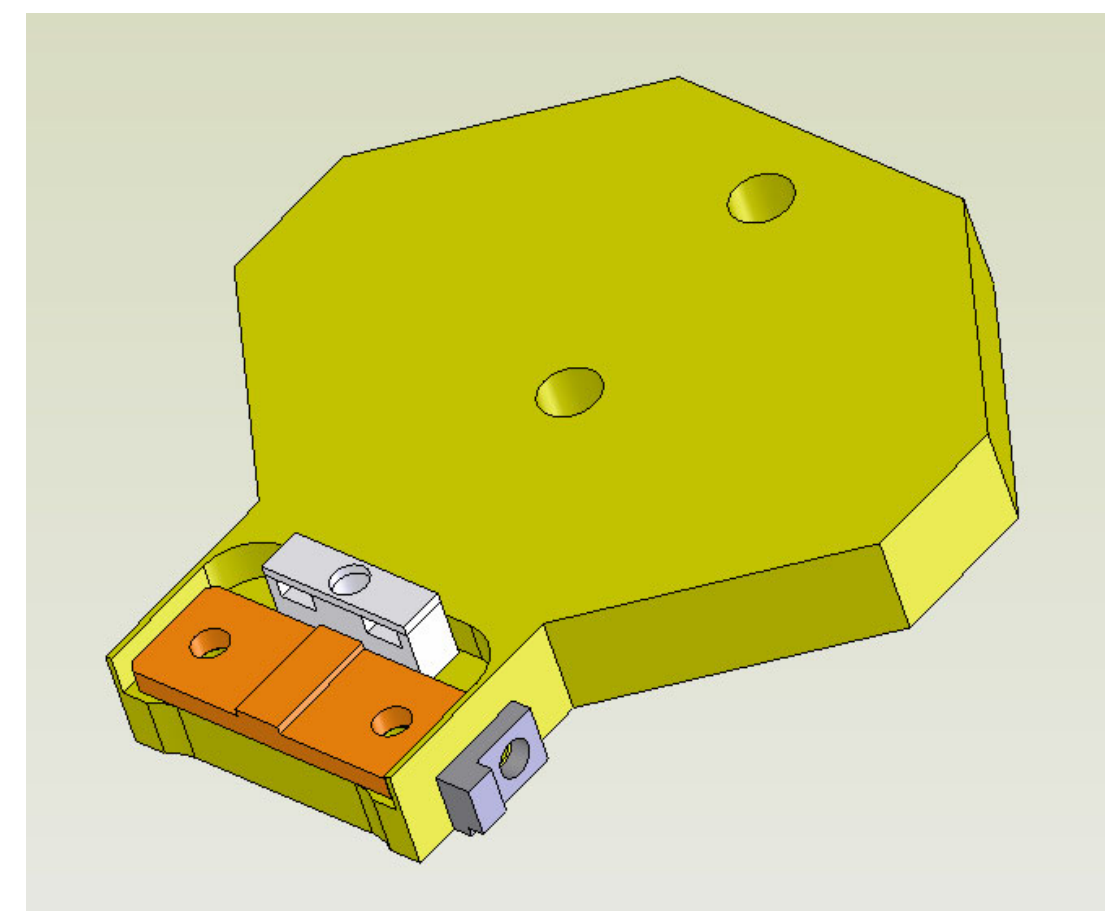

Figure 3.5. New QCL Mount Designed and Fabricated In-House, Showing Large Mounting Bracket (yellow/green), Laser Sub-Mount (orange) Corresponding Exactly to the ALPES Laser Mount to Facilitate Easy Interchange of Laser Devices, Mounting Clamp for electrical Connections (light gray) and Temperature Sensor (dark gray). There is a Minco Heater placed underneath the mounting plate directly under the QCL to minimize thermal path.

\subsection{Vacuum Systems, Manifolds, and Purging Requirements}

The experiments conducted in FY02 and FY03 used a simple vacuum system, minimal connections, and no purging facilities. Only two analytes could be used at any one time, and care had to be taken in order to avoid system contamination. This was not a problem in general because of the nature of the research being conducted at that time. However, moving towards a fieldable unit and testing more gases, some of which are hazardous, it has become necessary to design and build a custom mixing manifold, complete with purging system. This system is shown in Figure 3.6.

The manifold consists of a commercially available vacuum " $T$ " shown in Figure 3.6 in blue, with modifications to allow mixing of up to five analytes and one purging with inert gas such as dry nitrogen. An important aspect of the manifold design is to minimize interior surface area while allowing for effective pumping of gasses by the vacuum pump. Direct connection to the manifold is made via three techniques: vacuum tight precision welding, oxygen-free copper (OFHC) sealing flanges (Conflat type), 
and OFHC copper sealing tube connections (VCR type). These attachment techniques are highly reliable in terms of leak tightness and provide all metal surfaces to facilitate purging and baking if necessary. Other techniques may be used in parts of the system not directly connected to the mixing manifold. Not shown here, is the purging attachment to the optical cavity mounted on the optical bench, and the flexible line connecting the optical cavity and the pump. Purging can now occur in the cavity, or only the pumping line if required, or only the mixing manifold, allowing sticky molecules such as ammonia to be selectively removed from various components.

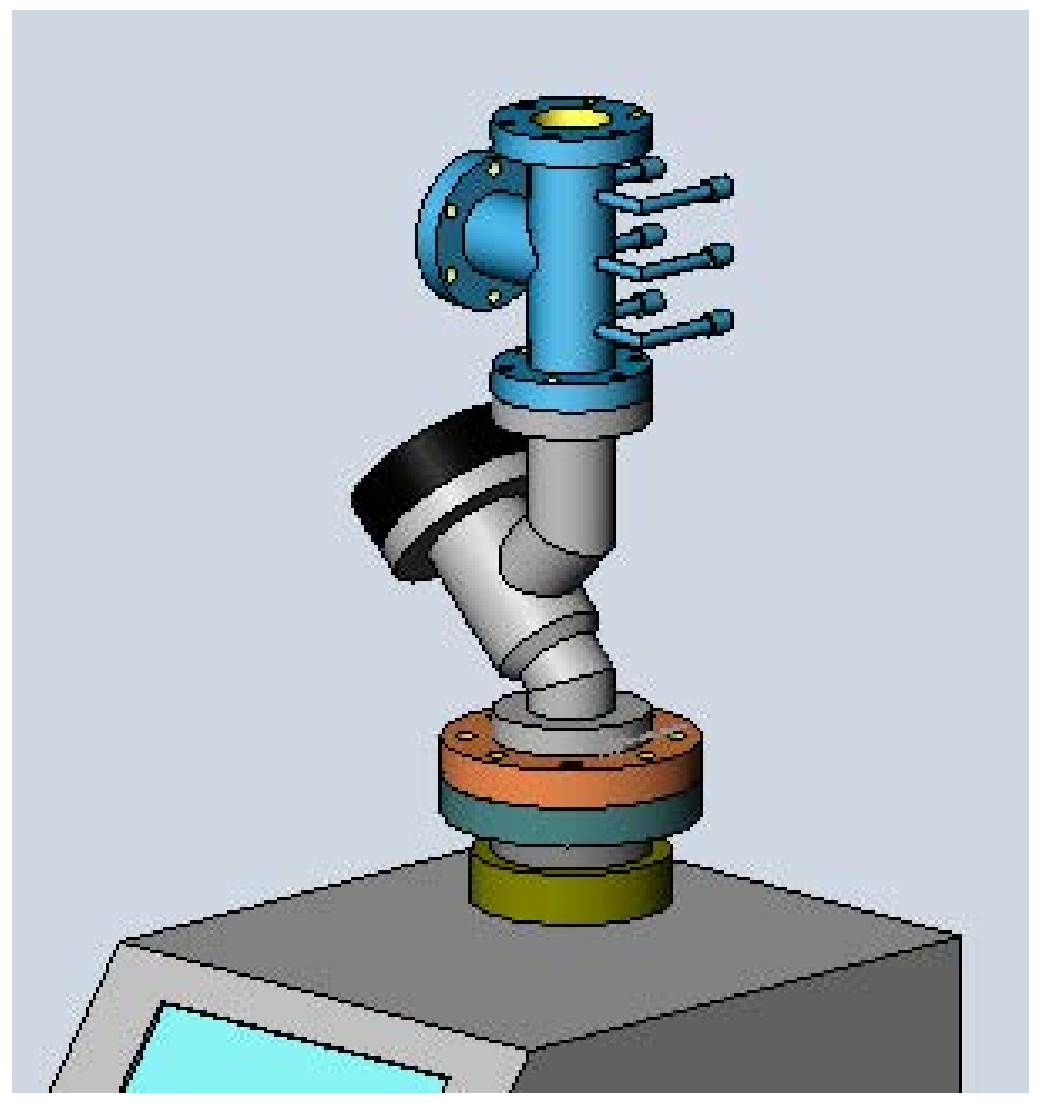

Figure 3.6. View of Top of Leybold Pumping Station with Mixing Manifold Shown in Blue. Six inlets are shown, allowing five different analytes and one purging gas to be connected. 


\subsection{Optimization of QCL Current Supply}

One of the major achievements in work in FY02 was the successful implementation of a low noise QCL current supply. The result of the low noise currents of this controller (currently at $10 \mathrm{nA} / \mathrm{Hz}^{1 / 2}$ at $10 \mathrm{~Hz}$ ) was to reduce the free running laser line-width down to roughly $100 \mathrm{kHz}$ from over a megahertz. (Typical "low noise" power supplies on the market have current noise of a few microamperes.) This linewidth is of the order of the transit time broadening seen inside the optical cavities we use for the LWIRCES, the beam being about a millimeter in cross section; i.e., about the same size as the observable homogeneous transition line-width, which makes the combination of the QCL and this current supply a formidable spectroscopy tool. This low noise level also makes the controller a valuable piece of intellectual property. Nevertheless, several possible improvements have become evident over the last two years. In the present configuration, the output stability over long periods is not as good as others on the market, there is a tendency of the regulator block to oscillate, and despite the low noise values there is still excess noise in certain parts of the units. The combined demands placed on the design of the QCL current controller for this and other projects, results in a difficult task for the designer. Not only does the QCL have to be low noise, it must be stable in the long term, resulting in minimum drift of the current over a period of minutes or hours. Additionally, when required the current supply must be able to sweep over a considerable proportion of its operating current at rates up to tens or even hundreds of $\mathrm{kHz}$. This is an unusually demanding set of constraints, resulting in a design that has been progressively modified over the last few years to gradually fulfill all the demands placed on it, which have also evolved during this time. In order to rectify the nagging problems listed above, each block of the unit was thoroughly examined in breadboard form, resulting in redesign of the regulator core, and other parts of the unit. A simplified schematic is shown in Figure 4.1.

The QCL is driven via MOSFET Q, which pulls QCL current through inductor L and passes it through resistor $\mathrm{R}$, to the negative rail labeled $-\mathrm{V}$. Amplifier A varies the drive to $\mathrm{Q}$ such that the voltage across the current sense resistor $\mathrm{R}$ is equal to the reference voltage $\mathrm{Vr}$. This results in a tightly regulated current through $\mathrm{R}, \mathrm{Q}$, and $\mathrm{L}$, and hence through the $\mathrm{QCL}$. The reference voltage $\mathrm{Vr}$ is developed across reference resistor $\mathrm{Rr}$, which is in turn determined by the current Ir, which is again in turn determined by the variable voltage source Vs and associated resistor Rs, in addition to any signal arriving from the Sweep Input via resistor Rv. The arrangement labeled Cascode Block 1, enables signals to be added at a virtual earth and then translated to another voltage level, in this case the $-\mathrm{V}$ rail, without interference from noise on this voltage rail relative to ground. This allows signals referenced to ground, such as signal generators and computers for example, to effectively interface with the current regulator, which operates referenced to the $-\mathrm{V}$ rail. The regulator arrangement consisting of $\mathrm{A}, \mathrm{Q}$ and $\mathrm{R}$, has a frequency response out to a several megahertz, allowing rapid sweeping of the QCL current, at full depth up to about $100 \mathrm{kHz}$. Faster signals out to around $70 \mathrm{MHz}$ are applied to the QCL through the Fast Input via Rf, and are referenced to ground via Cascode Block 2. The current source provides a bias Ib to allow bipolar signals to be applied via the Fast Input. 


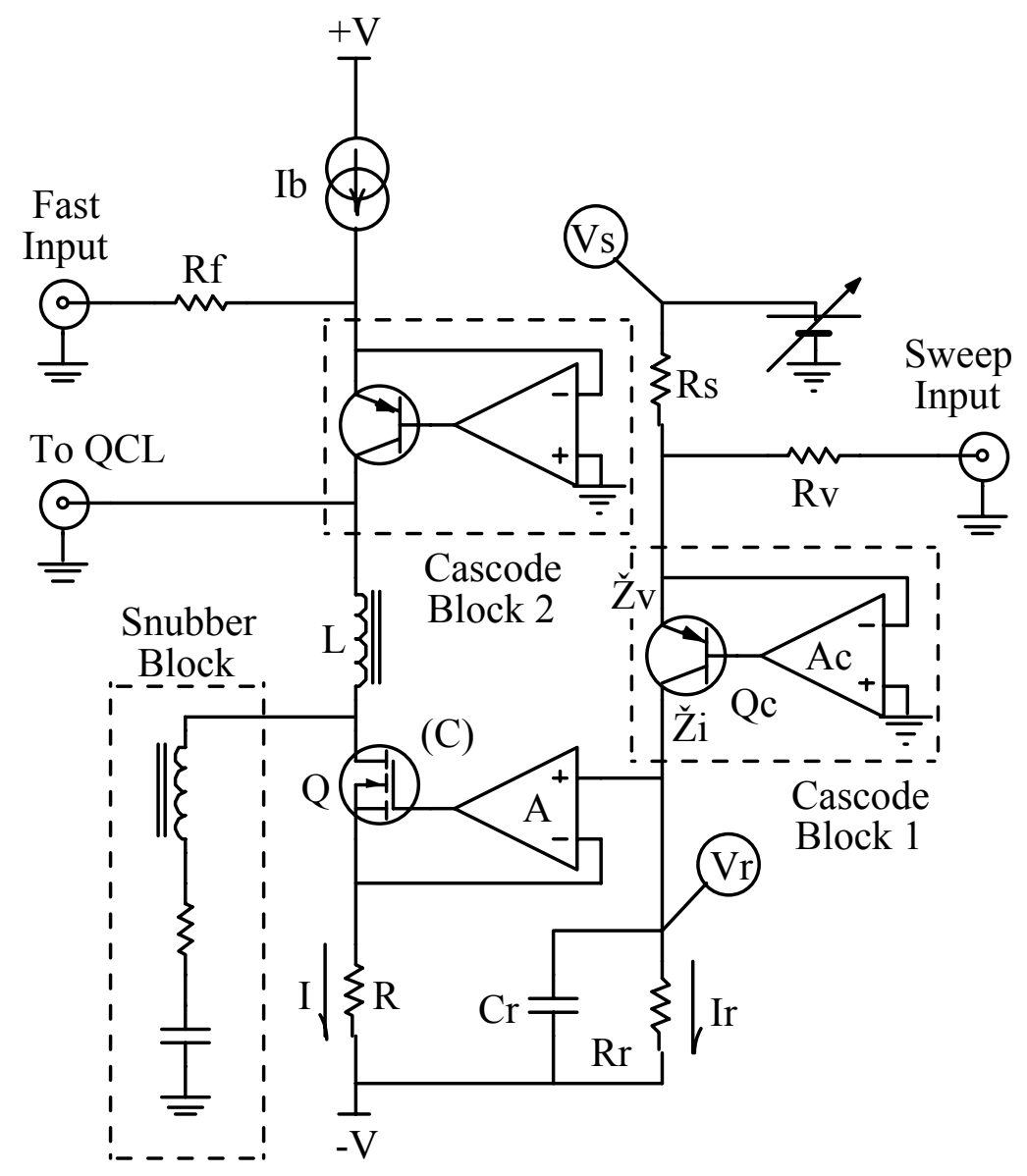

Figure 4.1. Simplified Schematic of the QCL Current Controller. The core of the unit is the regulator consisting of amplifier A, MOSFET Q, and current sensing resistor R, the latter two passing current $\mathrm{I}$. The reference for this regulator is provided by voltage source Vs and resistor Rs, via Cascode Block 1, and reference components $\mathrm{Rr}$ and $\mathrm{Cr}$. High frequency signals are passed into the QCL via Cascode Block 2, and are prevented from entering into the regulator core by inductor $\mathrm{L}$. The resulting resonance between $\mathrm{L}$ and the MOSFET capacitance C, is squelched by the Snubber Block.

\subsection{Stability of Main Regulator and Cascode Blocks}

In order that a regulator of this type be precise, the amplifiers (such as A in Figure 4.1) need to have high gain. A resulting issue can be marginal stability of the regulator, with small oscillations appearing at high frequencies often far beyond the desired operating bandwidth. Moreover, these oscillations are hard to study because they appear differently in different units, and not at all in others. A solution to this problem where high operating bandwidth is not an issue, is to reduce the gain of the amplifier at high frequencies by using capacitors around the amplifier A and transistor Q. In our case however, adding sufficient capacitance to reliably remove these oscillations in all units under all load conditions, greatly reduces the gain and operating bandwidth of the design. Through careful experimental work using a 
breadboard of only the regulator block to ensure no interference from other parts of the circuit, a reliable design with high gain and bandwidth was achieved without any oscillation regardless of the load applied to the regulator. This involved trying several and selecting the best operation amplifier (op amp) for the task of amplifier A, specific placement of small-valued resistors, and strategic use of ferrite beads to damp high frequency oscillations.

A similar problem existed on both Cascode Block 1 and 2; small oscillations at high frequency, which varied in manifestation from unit to unit despite being built on the same circuit board layout and using the same part numbers. This problem was solved in exactly the same manner as those in the regulator block. However it should be pointed out that the best amplifier, best resistor values, and ferrite bead placement for best results in the cascode blocks were different from those for the regulator block, probably due to the different nature of the transistors involved in each case.

\subsection{Noise of Cascode Blocks: A Question of Which Transistor}

Despite the already low noise level of the current controllers already in service (around $10 \mathrm{nA} / \mathrm{Hz}^{1 / 2}$ at $10 \mathrm{~Hz}$ ), the noise levels are still considerably in excess of the theoretical limit, calculated to be approximately $2 \mathrm{nA} / \mathrm{Hz}^{1 / 2}$. It was found that this discrepancy was due to excess noise in Cascode Block 1 and in the regulator block. Preliminary measurements of controllers where these blocks have been modified, exhibit noise levels down to $3 \mathrm{nA} / \mathrm{Hz}^{1 / 2}$, much closer to the theoretical value. Referring to Figure 4.1, Cascode Block 1 consists of a transistor Qc and an amplifier Ac, and operates in a similar manner to the regulator block, except that the reference voltage is at ground potential. Amplifier Ac regulates emitter voltage of Qc to ground, providing the virtual ground discussed earlier. A transistor exhibits both voltage and current noise, as a varying function of its collector current, shown here as $\partial \mathrm{v}$ and $\partial \mathrm{i}$ respectively. It can be shown using feedback theory that the action of the amplifier in the cascode block (in the limit of high gain) removes the effects of the transistor voltage noise, replacing it with its own input voltage noise. On the other hand, the current noise of the transistor, chiefly due to both shot noise and $1 / \mathrm{f}$ noise of the base current, is forced by the same feedback action to appear as differential noise between the emitter and collector currents of the transistor. Thus the current noise of cascode transistor Qc appears as additional voltage noise across Rr and thus across $\mathrm{R}$. This is turn, results in extra current noise through the QCL. Realizing this, and also that the small signal transistors from which we had chosen didn't have well documented current noise especially at low frequencies, we were prompted to test the output noise of the cascode configuration for various transistors, and from this we could estimate the transistor current noise after backing out factors such as Johnson noise of the resistors in the circuit, power supply noise, and voltage noise of the op amp. Figure 4.2 shows a comparison between the measured current and voltage noise of the NPN transistors $2 \mathrm{~N} 2222 \mathrm{~A}$, and $2 \mathrm{~N} 3904$. The current noise is clearly vastly different while the voltage noise is similar. The shot noise floor of the base-current of the 2N3904 is also shown, as a lower limit for this transistor; its measured noise approaches this value at around $1 \mathrm{kHz}$. The current noise of both transistors exhibit a $1 /(\mathrm{f})^{1 / 2}$ at low frequency, which is not atypical. 


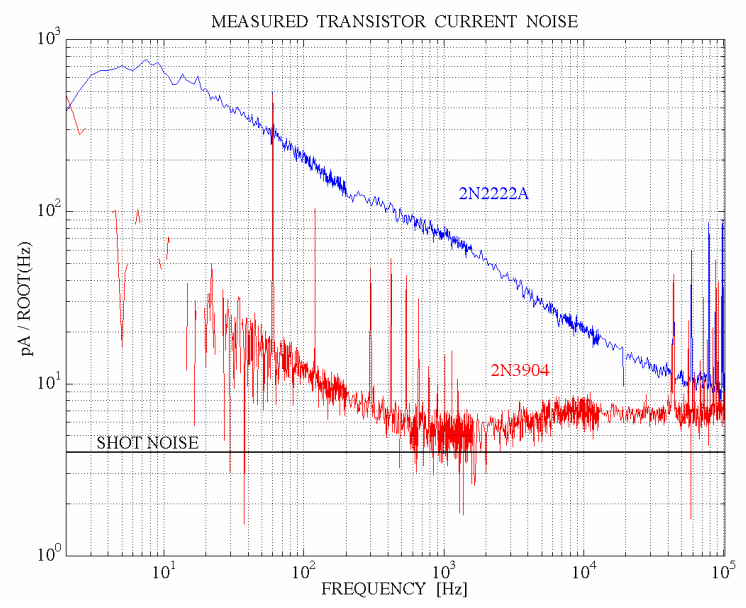

Figure 4.2a. Current Noise of 2N3904 (red) and 2N2222A (blue), the Latter Being More Than an Order of Magnitude Greater; Measured by Comparing Noise in Collector and Emitter Arms of the Breadboard Cascode Block Similar to That Shown in Figure 4.1

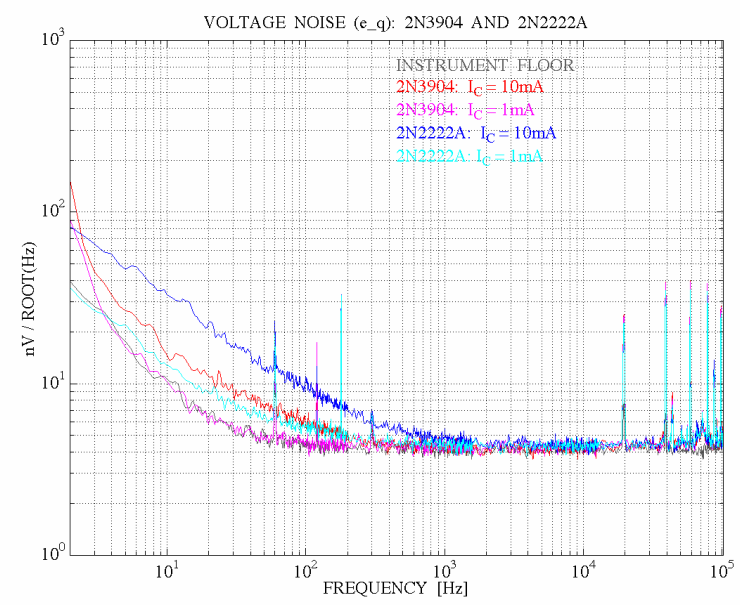

Figure 4.2b. Similar Voltage Noise Levels of 2N3904 (red and magenta) and 2N2222A (blue and cyan), for $10 \mathrm{~mA}$ and $1 \mathrm{~mA}$ Collector Currents as Indicated in the Legend, Measured at Emitter with Base Grounded

The result of the higher current noise in the collector arm of the cascode configuration when using the $2 \mathrm{~N} 2222 \mathrm{~A}$ rather than the $2 \mathrm{~N} 3904$, is that any voltage developed across a resistor in this path develops accordingly more voltage noise across it. This is illustrated in Figure 4.3, which shows voltage noise levels developed across $1 \mathrm{k}$ resistors in the emitter (blue) and collector (magenta) arms in the cases of both transistors in question. Again it is clear that the case of the $2 \mathrm{~N} 2222 \mathrm{~A}$ is approximately an order of magnitude worse at frequencies below a kilohertz. The voltage noise across resistors in the collector arm of such a cascode circuit will always be worse than that across similar resistors in the emitter arm. This is because of the presence of Johnson noise in both resistors coupled with the servo action of the amplifier, which results in suppression of the emitter-resistor noise, and its subsequent transference and addition to that already in the collector-resistor. This is a principal reason for the remaining difference between the traces in Figure 4.3a. Others, particularly at the higher frequency end of the graph, include inaccuracies in our analysis due to the subtraction other converging noise traces, such as power-supply noise.

As in-depth data on the current noise statistics of these transistors is not readily found, as it is usually the voltage noise that is considered in many designs. Also, since the $2 \mathrm{~N} 2222 \mathrm{~A}$ and its complement 2N2907 have higher current capacity, they were used in the designs of our previous versions of the current controller. Clearly an order of magnitude better noise performance is to be gained by switching to the 2N3904 and its complement 2N3906. The older versions are also being retrofitted where lower noise performance is imperative. 


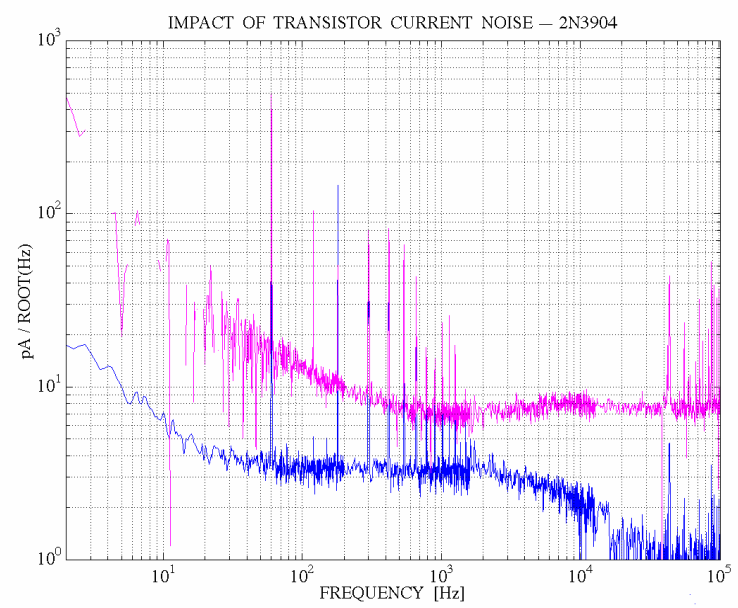

Figure 4.3a. Voltage Noise Across $1 \mathrm{k}$ ohm Resistors in the Emitter and Collector Arms of the 2 N3904 Cascode, Blue and Magenta, Respectively

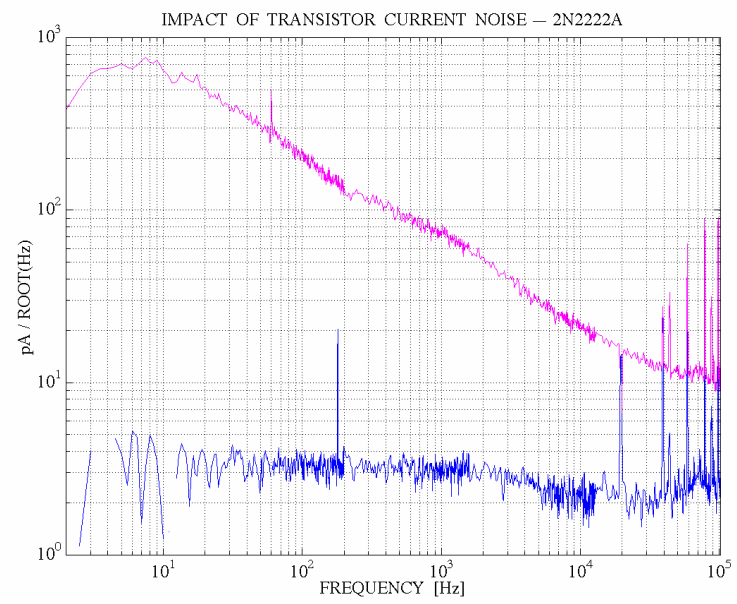

Figure 4.3b. Voltage Noise Across $1 \mathrm{k}$ ohm Resistors in the Emitter and Collector Arms of the 2N2222A Cascode, Blue and Magenta, Respectively

\subsection{Long-Term Stability and Reference Drift}

The long-term stability of a QCL current supply can be of primary importance if there is no other mechanism of stabilization. Until now, long-term absolute current stability has not been of primary importance, although it was assumed that the units would be more than adequately stable for QCL operation. It was discovered however that the current could drift up to $5 \mathrm{~mA}$ or so over the course of an hour. The discovery of drifts in the references that were used to both obtain the set voltage Vs in Figure 4.1, and the bias current $\mathrm{Ib}$ for Cascode Block 2, provided more than adequate explanation for this. Precision references and current sources are used in this design revision. Also, additional cascode blocks in later units such as the QCL servo are being eliminated, removing extra source of QCL current drift. Preliminary measurements of the latest controllers show noise drifts of less than of the order of 200 parts per million, a vast improvement over the above drift. The further addition of precision resistors at key locations in the circuit will improve this even further.

\subsection{Output Filtering, Resulting Resonances}

It was mentioned in the outline of operation of the controller that fast signals out to tens of megahertz are applied to the QCL via the Fast Input (Figure 4.1), and are referenced to ground via Cascode Block 2. The MOSFET Q has an output capacitance of about $193 \mathrm{pF}$, which could shunt these high frequency signals straight through to the $-\mathrm{V}$ rail. This is prevented by the presence of inductor $\mathrm{L}$, which exhibits high impedance to high frequency signals. While this presents no operational problems to the regulator performance, there is a resulting series resonance between inductor $\mathrm{L}$ and the capacitance of the MOSFET. This causes a large notch in the response of the Fast Input, and a phase shift that could cause problems with servo control of the QCL in particular. In retrospect, this was one probable reason why the servo design for the QCL frequency stabilization was particularly challenging. 
After some experimental tests and numerical modeling, it was found that this resonance could be removed with minimal disturbance to the circuit operation by the addition of the Snubber Block, consisting of a series resistance, capacitor and inductor. Figure 4.4 shows experimental measurements showing such a resonance in the response of the Fast Input of a breadboard version of the regulator block, and its removal by the addition of a suitable snubber network. This improvement will allow better high frequency drive performance to the QCLs necessary for effective locking and modulation.

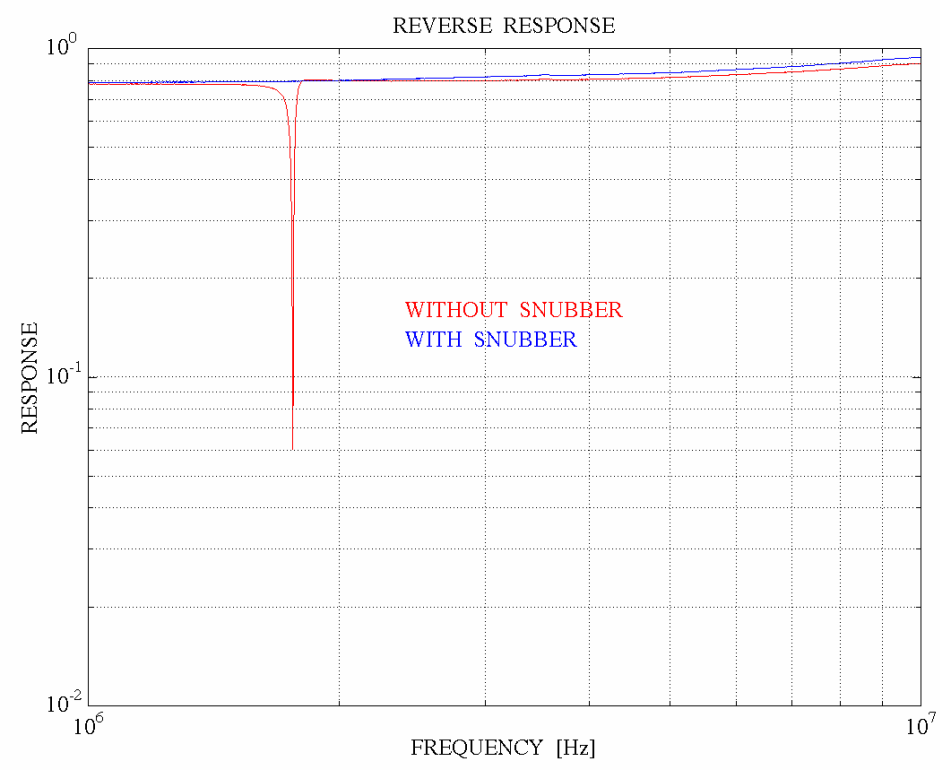

Figure 4.4. Transfer Function of Fast Input Network. Red trace shows resonance of output inductor $(\mathrm{L}=42 \mu \mathrm{H})$ with the MOSFET output capacitance $(193 \mathrm{pF})$. Blue trace shows response with snubber network, the resonance completely removed. 


\subsection{Plans for FY05}

In addition to examining sensor performance for a range of test analytes as discussed in Section 2 above, work during FY05 will be in three specific areas: optimizing the FM dither technique to operate with Doppler-broadened features rather than Lamb dips, automating the cavity locking technique specifically to accommodate dropouts from lock, and reducing the overall footprint of the sensor to ready it for field testing. For reference, Figure 5.1 shows a schematic of the FM Recovery Sensor highlighting in green areas of focus for FY05, including the cavity mirror mount and piezo assembly used for the dither modulation, the stabilization electronics, and certain optical components that are either particularly sensitive or bulky.

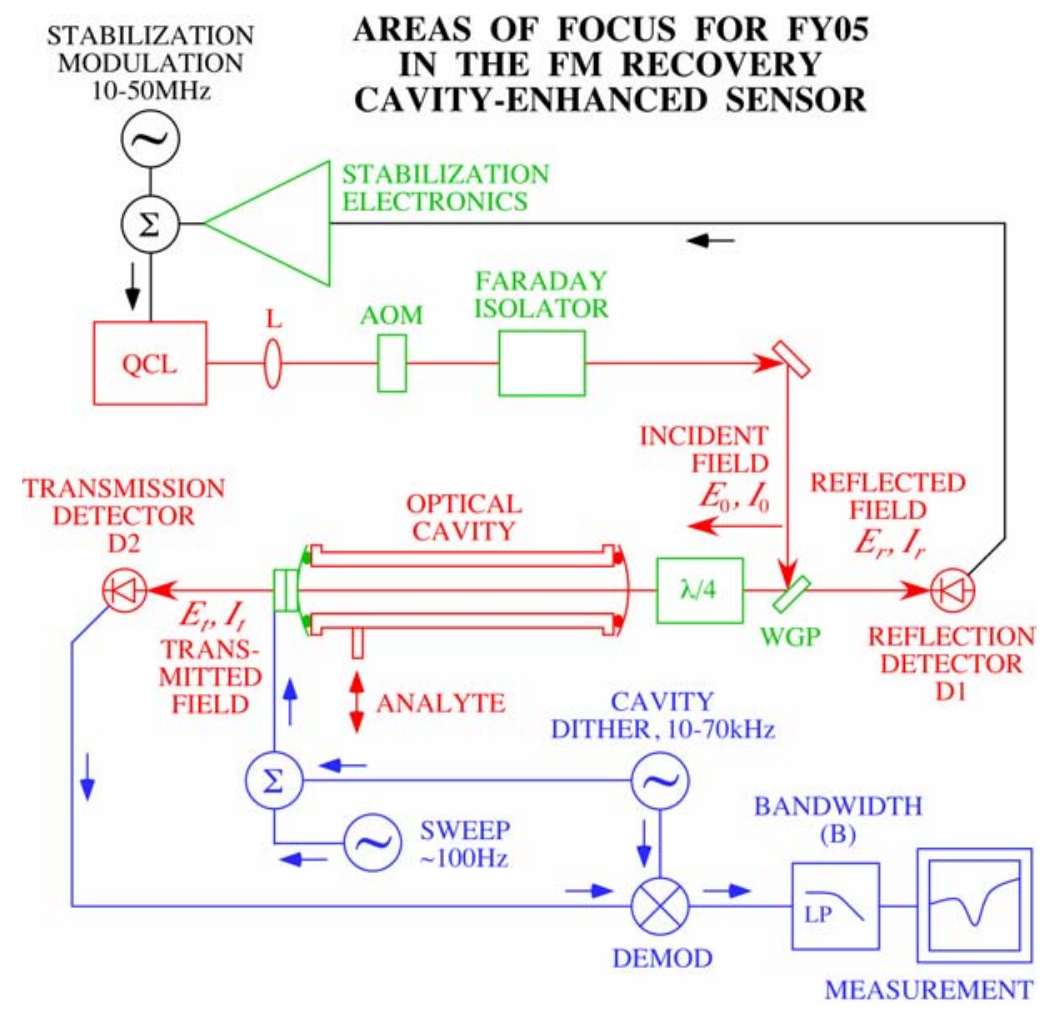

Figure 5.1. FM Recovery Cavity-Enhanced Sensor, Reproduced from Figure 1.2, But With Areas of Focus for FY05 Shown in Green. The QCL is locked to the cavity as before. However, in addition to the cavity length being scanned, it is also dithered or modulated. The transmission signal from D2 is demodulated accordingly, then filtered and recorded as before. Intensities and electric field amplitudes are indicated for incident, reflected and transmitted fields. 


\subsection{Optimizing FM Dither Technique}

Until now the cavity dither depth has been made to correspond to that of the Lamb dips seen in our experiments, this depth being at most several megahertz. In order to be optimized for Doppler-limited profiles, this depth will have to be increased to a significant portion of the Doppler width, which for example is about $70 \mathrm{MHz}$ for nitrous oxide at $297 \mathrm{~K}$. In the current configuration represented in Figure 5.2, a piezo disk pushes against a mirror, which in turn rests against a vacuum O-ring, forming at once a seal between the mirror and the cavity housing, and a compressible seat that allows the mirror to move slightly under the action of the piezo. The problem with this design is that this movement is limited as the O-ring is already considerably compressed by the pressure differential between the room and the interior vacuum.
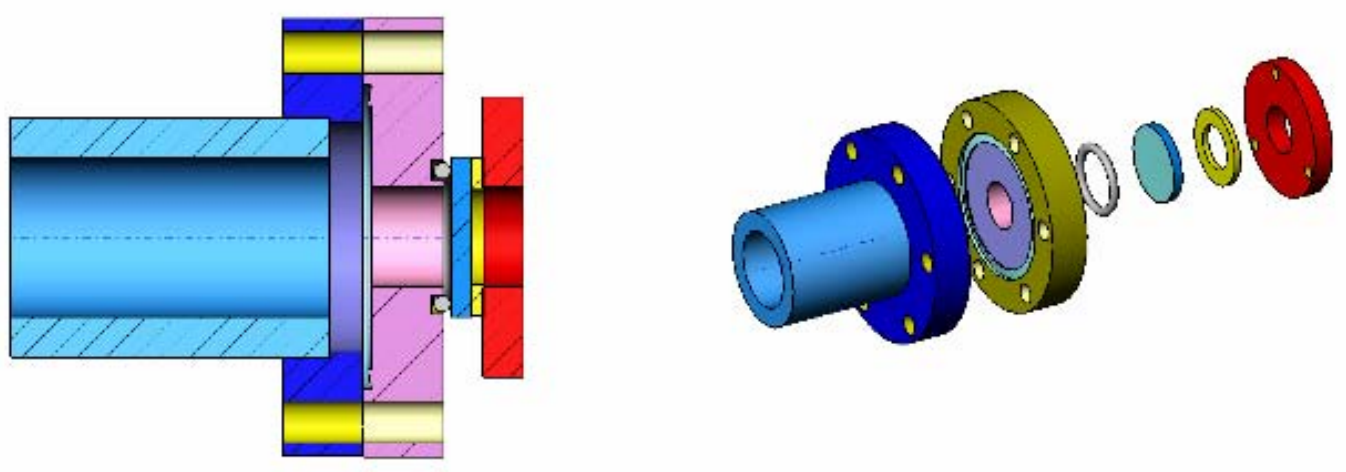

Figure 5.2. Cut-Away and Exploded Views of the Present Piezo and Moving Mirror Mount Used to Produce the Cavity Dither Modulation. At present, the annular piezo disk (yellow) is clamped against the mirror (blue) seated against an O-ring (gray), in turn seated in a groove in the vacuum flange.

Depicted in Figure 5.3 is a new design currently being built in which the mirror and piezo arrangement is entirely internal to the housing, and a window (not shown) is used to make the vacuum seal. In this case, the mirror will can move more freely, and attain greater excursions allowing deeper dither modulation depth.

\subsection{The Next Step in Stabilization}

The next area targeted for optimization is the stabilization electronics, which locks the QCL to the optical cavity. While the performance of this system is excellent in that it holds the laser and cavity together with a precision down to a fraction of a Hertz, it is currently not automated. Consequently, the specific cavity mode required has to be selected manually, and the various stages of gain activated in sequence to lock to a specific mode. A computer can perform the tasks of adjusting gain and turning on various parts of the servo, however, specific changes to the electronics are required to implement this. Moreover, there is the problem of optical interference with the lock that needs to be addressed. Due to 

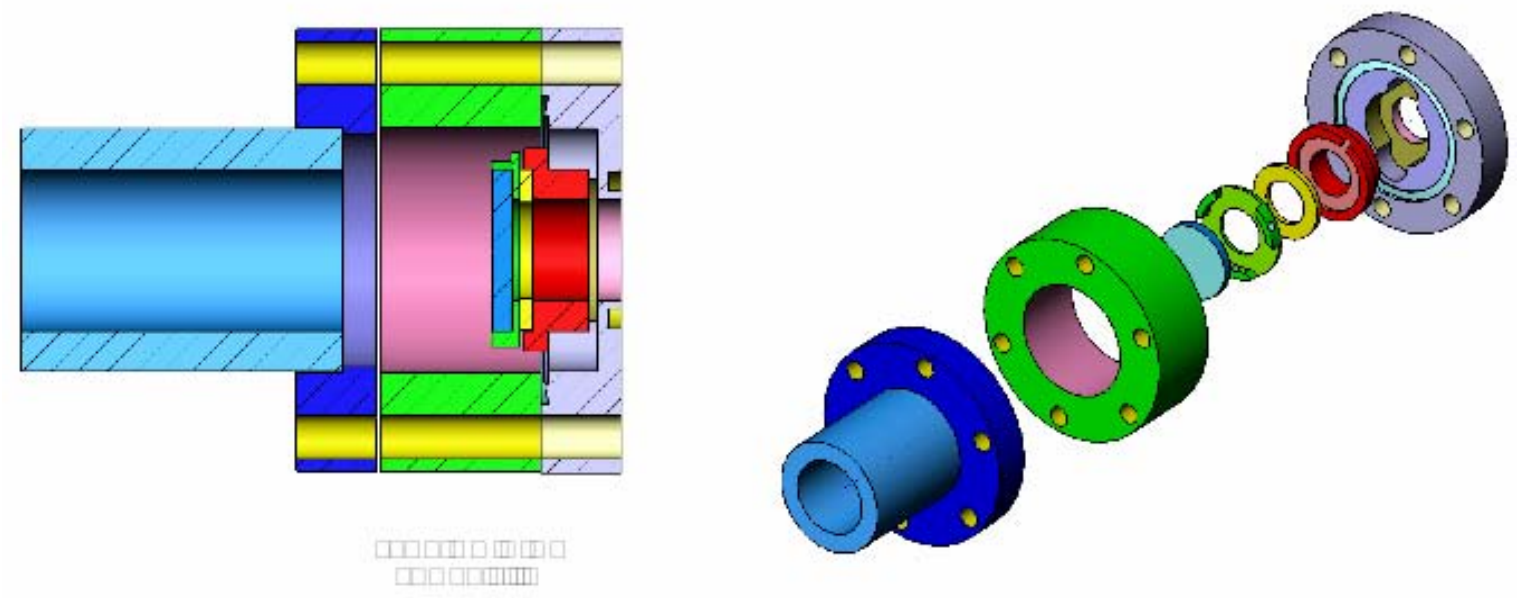

Figure 5.3. Cut-Away and Exploded Views of the New Piezo and Mirror Mount Design, Which is Internal to the Low-Pressure Gas Cell. The O-ring remains external and will now be in contact with a BaF window (not shown) rather than the mirror in order to form the vacuum seal. The mirror (blue) is mounted in a thin aluminum cup (green), glued to the annular piezo disk (yellow), which is in turn glued to a macor ceramic insert (red) for electrical insulation, which is screwed into the vacuum flange. An electrical feed-through (also not shown) is now needed to pass the signal to the piezo disk inside the chamber.

the optical improvements seen to the system over the last several years, one of the down sides has been an increase in optical feedback to the QCL despite several stages of isolation. A consequence is random and sudden jumps in laser frequency, causing almost instantaneous loss of lock. This situation is exacerbated by otherwise essential components of the electronics known as integrators, which in the event of the loss of cavity lock; usually throw the laser completely to one side or the other of the frequency control range. This is a common problem in stabilization systems, and there are many different solutions, none of which are universal. The principal goal of the optimization of the stabilization electronics in FY05 is to be able to deal with such frequent and sudden dropouts, and being able to return the laser quickly to the previous operating frequency. To our knowledge this hasn't been achieved before, so the development of a reliable system to do this will represent a significant achievement.

\subsection{Reducing the Footprint, and the Option of a Ring Cavity}

Several optical components in the current bench top experiment are quite large. These include the Faraday Isolator, the Acousto-Optic Modulator (AOM) and the quarter-wave rhomb ( $\lambda / 4)$. During FY05 we will explore methods of reducing the size of the Faraday Isolator and eliminating altogether the AOM. The reason for their size is principally due to the inherent inefficiencies of these devices when operated in the infrared. Modulation indexes, electro- and magneto-optic, and acousto-optic, are far less in the LWIR than in the visible and NIR. Consequently, such devices require higher magnetic or electric fields, or higher drive power. This is not better illustrated than in the case of the AOM. It's visible wavelength counterparts are much smaller, and require a Watt of power if that, while the device we use for the LWIR is an order of magnitude larger and heavier, and requires water cooling and 70 Watts of RF power. 
However, the decision to use Doppler features rather than the sub-Doppler Lamp dips as the principle feature of choice, allows the use of a ring cavity rather than a linear cavity, which could simplify the layout and reduce the footprint. It is not possible to see Lamb dips in a ring resonator, because of the lack of counter-propagating beams responsible for the appearance of sub-Doppler features. Dopplerbroadened features remain unaffected by this difference.

The use of a ring cavity instead of a standing wave cavity could possible give much less back reflection, thus alleviating the need to have two stages of isolation, allowing us to dispense with the AOM. It will also allow us to dispense with the bulky $\lambda / 4$ rhomb and sensitive and fragile wire grid polarizer (WGM) shown explicitly in Figure 5.1. These two components were not shown in the earlier Figure 1.4 for simplicity, but are relevant to this discussion. Together, they form an "optical circulator", allowing the maximum possible coupling of the input field to a linear cavity, and also of the cavity reflection to the reflection detector. This is achieved by rotating the polarization from linear to circular after reflection from the WGP, and then back to linear of the opposite orientation upon reflection from the cavity, so that this reflected field passes through the WGP on to the detector. The reason why a ring cavity makes the above simplifications possible is clear from Figure 5.4, which is a version of the FM Recovery Sensor based on a ring cavity.

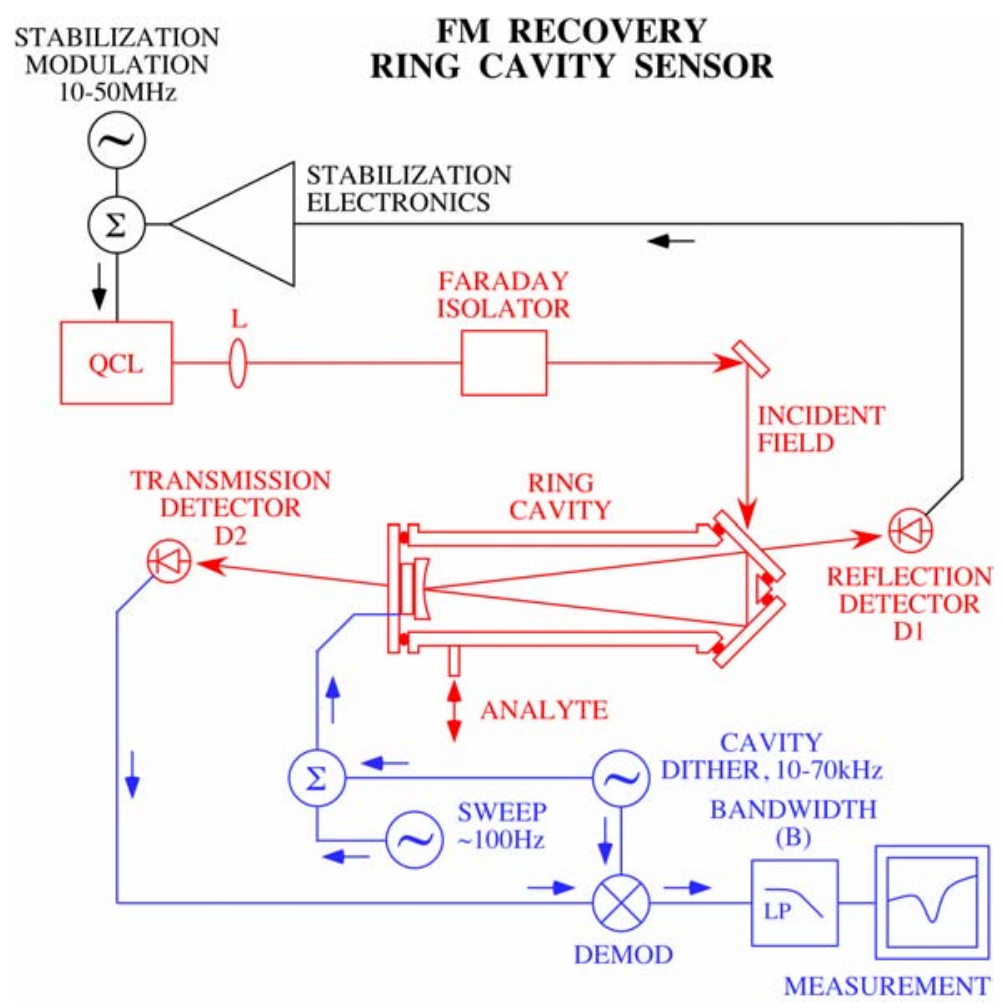

Figure 5.4. Ring Cavity Version of the FM Recovery Sensor. The architecture of the ring cavity could in principle allow much simplification and reduction of the footprint. Note that the AOM, $\lambda / 4$ and WGP of Figure 5.1 are missing! 
The incident and reflected fields from the input mirror are no longer coincident due to the circular optical path within the ring resonator. Consequently, the reflected field from the optical cavity no longer reflects directly along the incident path and possibly back into the laser causing optical feedback and possible damage. As a result, the amount of optical isolation required is reduced, hopefully allowed the AOM to be removed. As this device requires water-cooling and 70 Watts RF power derived from a large amplifier unit mounted under the table, its removal would represent a considerable change to the weight and power requirements of a fieldable package. Some isolation is still required however, as light reflected from components after cavity transmission, for example, the transmission detector or its focusing lens (not shown), will couple directly back through the ring cavity and back up the incident path.

Consequently the Faraday Isolator is retained. Despite its present size and weight, it requires no external connections to power or water, and hence is desirable over the AOM. The size of the Faraday Isolator is due to the requirement for a large and uniform magnetic field (developed by permanent magnets) to interact with a small crystal optic at the center of the device. It is hoped that through judicious searching and in-house testing that a smaller unit can either be either bought or made during the next twelve months. 


\subsection{Summary and Outlook}

The selection of a specific sensor architecture represents a new phase of experimental research and development. Until now, the focus of the experimental work was to demonstrate and explore the performance of the various architectures, and to identify and quantify the limitations of each technique. A major part of the work conducted in FY04 was to choose more strategic analytes, and molecular transitions, to implement better gas handling systems and modifying laboratory systems to accommodate these analytes. Several technical issues were addressed, that were important for fieldable sensor design, such as improving the QCL mounting system and thus its alignment stability within the cryogenic Dewar, and refining the drive electronics for the QCL. We are now at a stage where we can move forward with the selected technique of FM Recovery Cavity Dither, focus on optimizing the layout and optical components for minimum footprint, test key selected analytes, and finally begin to automate the sensor. With the move away from the resonant side band technique requiring matched cavity length, and away from Lamb dips, the larger linear cavity currently used could eventually be replaced with a smaller ring cavity once this architecture has been explored over the next twelve months.

Due to differences between the sizes and efficiencies of typical optical components in the LWIR, progress of the LWIR-CES has focused on resolving related issues rather than the immediate fielding of an LWIR-CES instrument. However, the potential advantages of this technology as implied by the difference between the near IR and LWIR spectra of typical analytes as seen in Figure 2.1, imply that any progress represents disruptive advances in sensor capability. It is projected that in two to three years, LWIR sensors based on the cavity-locked technology will be deployed in light weight, accurate, reliable packages, providing advanced warning to military and civilian personnel in the event of a chemical or nuclear attack. These sensors will also allow monitoring of facilities of interest to determine quantities

and types of emissions. Test results from the latest version of the QCL laser controller give $3 \mathrm{nA} \mathrm{Hz}^{-1 / 2}$ at $10 \mathrm{~Hz}$ current noise, which is several orders of magnitude lower than commercially available systems. This will result in laser line widths on the order of $\sim 30 \mathrm{~Hz}$ (to be measured in FY05) enabling higher signal to noise and resolution of the transmitted signal through a sample of interest. Particularly intriguing is the now facile ability to resolve isotopic species of interest using this high resolution in a single laser scan. Isotopic ratios of $\mathrm{CO}_{2}$ provide origin history for the source of the carbon and can distinguish biological sources from background geological sources. In the atmosphere the isotopic ratios of $\mathrm{HDO}$ and $\mathrm{H}_{2} \mathrm{O}$ provide dynamic information on the mixing of air over oceans and the rates at which these species are lofted over land. This information is important in the analysis of the plumes from explosions, chemical releases, and provides insight into global warming mechanisms. Isotopic ratios are also very important in many nuclear fuel production processes. Significant advances in the detector preamplifiers combined with the stable laser current supplies will permit detection of chemical species down to a level of $15 \mathrm{ppt}$ level (calculated in ammonia at 10 Torr). This ultra-sensitive detection limit will provide a superior technique to monitor chemicals of interest.

Continued development of this sensor using this technology continues to represent a significant improvement over existing techniques, such as Gas-Chromatograph Mass Spectrometry (GCMS). While offering superb sensitivity, GCMS has specific limitations because it does not distinguish based on the chemical behavior of a molecule, but rather the mass of its constituent atoms. Moreover, GCMS does not 
discriminate between isomers, or different atoms of the same mass. On the other hand the spectroscopic features of a gaseous species are highly specific and reliable indicators. Much greater selectivity is thus available with techniques using spectroscopy. There are also many practical issues that make GCMS systems difficult to field, which even the current cryogenic LWIR laser systems don't suffer from. The GCMS has long response times (>70s) while the LWIR laser systems can provide near instantaneous measurements $(<1 \mathrm{~s})$. Detectors used in GCMS systems often include radioactive sources which complicates shipping and deploying the instrument, especially to sensitive international sites. The radioactive sources also tend to drift with changes in temperature and pressure which requires constant calibration with zero air using complicated computer controlled gas handling systems, heavy gas cylinders, and heavy power hungry pumps and solenoids. This calibration sequence leads to gaps in data streams which could lead to delays or missed detection opportunities. The pumps and solenoids often generate mechanical and electrical noise which interferes with other instruments. GCMS systems are not useful for reactive species such as $\mathrm{HCl}, \mathrm{HF}, \mathrm{HI}, \mathrm{HBr}, \mathrm{NH}_{3}, \mathrm{~N}_{2} \mathrm{H}_{4}$, etc. The columns require constant maintenance and must be baked out to remove contaminants resulting in decreased duty cycle. In fact the cleaver terrorist could release a reactive gas to "poison" the GCMS columns and render the instrument inoperative. The LWIR laser system in contrast can provide rapid response time, high duty cycle, and very high sensitivity with near zero false positive readings. A fieldable version of even a cryogenically operated LWIR CES could weigh less than $20 \mathrm{~kg}$, low power consumption $(<50 \mathrm{~W})$ system whereas GCMS systems are heavy $(>60 \mathrm{~kg})$ and require high power $(>300 \mathrm{~W})$. When room temperature CW QCL operation becomes common-place, this footprint will decrease considerably. 


\subsection{References}

Gianfrani L, RW Fox and L Hollberg. 1999. J. Opt. Soc. Am. B, 16(12):2247.

Ishibashi C and Hiroyuki Sasada. 1999. Jpn. J. Appl. Phys. 38:920.

Ye J, L-S Ma and JL Hall. 1998. J. Opt. Soc. Am. B, 15(1):6. 


\section{Distribution}

No. of

\section{Copies}

\section{OFFSITE}

LTC Ariel Cuadrado

United States DOE

NNSA/NA-22

1000 Independence Ave. SW

Washington, DC 20585

Dr. Rhys M. Williams

United States DOE

NNSA/NA-22

1000 Independence Ave. SW

Washington, DC 20585

Dr. Michael Meier

United States DOE

NNSA/NA-22

1000 Independence Ave. SW

Washington, DC 20585

Dr. David Berry

United States DOE

NNSA/NA-22

1000 Independence Ave. SW

Washington, DC 20585

Mr. Ralph Hastings

United States DOE

NNSA/NA-22

1000 Independence Ave. SW

Washington, DC 20585

Mr. Eric Sanders

United States DOE

NNSA/NA-22

1000 Independence Ave. SW

Washington, DC 20585
No. of

Copies

Mr. W. Randy Bell

United States DOE

NNSA/NA-22

1000 Independence Ave. SW

Washington, DC 20585

Dr. Vaughn Standley

United States DOE

NNSA/NA-22

1000 Independence Ave. SW

Washington, DC 20585

LTC John C. Carrano, PhD

Program Manager

DARPA, MTO

3701 N. Fairfax Dr.

Arlington, VA 22203-1714

\section{ONSITE}

\section{Pacific Northwest National Laboratory}

$\begin{array}{ll}\text { Anheier, NC } & \text { K5-25 } \\ \text { Bruckner-Lea, C } & \text { K5-25 } \\ \text { Cannon, BD } & \text { K5-25 } \\ \text { Clemmer, RG } & \text { K8-29 } \\ \text { Dudder, GB } & \text { K8-29 } \\ \text { Harper, WW } & \text { K5-25 } \\ \text { Johnson, BR } & \text { K6-24 } \\ \text { Munley, JT } & \text { K6-24 } \\ \text { Myers, TL } & \text { K5-25 } \\ \text { Nguyen, VT } & \text { K5-10 } \\ \text { Scott, DC } & \text { K5-25 } \\ \text { Schultz, JF (26) } & \text { K5-25 } \\ \text { Stewart, TL } & \text { K5-25 } \\ \text { Strasburg, JD } & \text { K5-25 } \\ \text { Taubman, MS } & \text { K5-25 } \\ \text { Wojcik, MD } & \text { K5-25 } \\ \text { Information Release Office (7) } & \text { K1-06 }\end{array}$

Distr.1 